\title{
4. Congestion management and transmission rights
}

\section{Dmitri Perekhodtsev and Guido Cervigni}

\subsection{INTRODUCTION}

In this chapter we discuss the impact of electricity transmission technology on the design and outcome of electricity markets.

Electricity is transported on a transmission network from the place where it is generated to the place where it is used. Transmission congestion occurs when generation and demand schedules cleared in the market lead to a set of power flows violating one or more network constraints. When this happens, congestion must be alleviated. Electricity transmission is different from other goods and commodities for which transport flows can be re-routed relatively easily. On the contrary, increasing (net) injections at some locations and decreasing them at others is the primary and often only option to relieve congestion.

Market arrangements differ across electricity markets in the way they induce market participants to deviate from the injection/withdrawal levels they would implement in the absence of any transmission constraints. Two general approaches can be identified. The first restricts the transactions that market participants can enter into to those producing feasible power flows. This is achieved by allocating and enforcing a feasible set of rights to inject and withdraw power at the different network locations. As a result, in the event of congestion, demand and supply clear at different prices at different locations, reflecting the different costs of meeting the incremental demand for electricity at each location. Generally the market-clearing prices at import-constrained locations are higher than at export-constrained locations. This general approach has been adopted in many US electricity markets, such as PJM, New York, New England, California, Texas and the Midwest, as well as in New Zealand. In Europe this approach has been implemented in the markets of Norway, Italy, and recently Sweden, where several network nodes are grouped into market zones, and the set of feasible market transactions is the result of constraints on the net power flows between the zones. The ongoing projects 
aiming to integrate the European national spot markets also feature locational pricing, in which each participating country is treated as a market zone.

The second approach compensates market participants for deviating from the level of injections and withdrawals they would have selected in the absence of transmission constraints. This practice is termed 'redispatch'. In this approach the electricity market is initially run neglecting any transmission constraints. Subsequently, if the market outcome produces power flows that violate one or more transmission constraints, generators and possibly consumers are paid to modify the level of injections and withdrawals they have scheduled in the first unconstrained stage. This approach is widely adopted in the UK, France, the Netherlands, Spain and Germany.

In Section 4.2 we describe how net injections at the nodes of a transmission system produce flows over elements of the transmission network. In Section 4.3 we discuss congestion management methodologies based on restricting the transactions that market participants can enter into to those producing feasible power flows. In Section 4.4 we discuss the alternative approach, in which market transactions are not subject to any networkrelated restrictions, and congestion is relieved at a subsequent stage. In Section 4.5 we discuss an intermediate approach, in which network-related constraints to market transactions are defined at a zonal level. In Section 4.6 we address the longstanding policy debate on the relative merits of congestion management via re-dispatch and via locational price differentiation. In Section 4.7 we discuss alternative approaches to transmission network development.

\subsection{NETWORK EFFECTS AND LOCATIONAL DIFFERENTIATION OF THE VALUE OF ELECTRICITY}

When one or more network constraints are binding, injections at different nodes may not be substituted or can only be partially substituted to meet a given demand. Real-world transmission networks often have a complex topology featuring multiple loops and parallel paths connecting any two points in the network. In such networks, binding network constraints may create relations between transactions across different locations with varying degrees of complementarity or substitution. For example, if a transaction between two nodes is restricted by a transmission constraint, this constraint can be variously relieved by increasing net injections at some nodes of the network or by decreasing net injections at some other nodes. 
When network constraints are binding, the cost of meeting an incremental increase in demand can differ between nodes, as the least-cost way to meet the increased demand at different nodes may involve increasing the production of different generating units. In this section we analyse the effect of network constraints on the cost of meeting a net demand increase at the different network nodes, while in Sections 4.3, 4.4 and 4.5 we investigate how this feature of the electricity industry impacts on the market outcome, under alternative market designs.

In Section 4.2.1 we illustrate the relationship between the net injections at the nodes of a large transmission network and the flows over its elements, having made certain simplifying assumptions. In Section 4.2.2 we discuss how binding network constraints affect the incremental cost of electricity at different locations.

\subsubsection{Power Flows}

A transmission network can be considered as a set of power lines connecting nodes. Each node represents a substation, where generators and loads are connected to the network. A net injection at a node, at a given time, is the difference between the total production of all generators and total consumption of all loads located at this node taking place at that time. Power flows over each line of the network are determined by the net injections at all nodes as well as the network topology. The network topology is defined by the electric properties of power lines and the way they connect different network nodes. ${ }^{1}$

In power networks featuring alternating current (AC), the relationships between net injections and power flows over the transmission lines are not linear. However, in this chapter we use a linear approximation of these relationships, which is often called the 'DC (direct current) approximation'. We also abstract from transmission losses in the network. Such a model represents a realistic approximation that is often used to analyse the effects of loop flow and network interactions. ${ }^{2}$

Under the DC approximation, neglecting transmission losses, the power flow on each network element brought about by a set of net injections is determined by the following properties:

- Superposition Power flows produced by a given set of net injections can be computed as the sum of flows produced by any combination of balanced net injections in which the initial set can be broken down.

- Least resistance path The share of power that flows along each network path between each source node and sink node is inversely 
Table 4.1 Net injections (MW)

\begin{tabular}{lc}
\hline Node & Net injection \\
\hline 1 & 6,000 \\
2 & 1,500 \\
3 & $-7,500$ \\
\hline
\end{tabular}

proportional to the relative resistance of such a path: the lower the resistance of a path the larger the share of power that flows along that path.

Table 4.1 and Figure 4.1 illustrate these properties and show how they can be used to compute the power flows brought about by a given set of injections and withdrawals. We considered a simple triangular network with three nodes connected by three identical lines. Such a network is the simplest example of a network with parallel paths and can be used to illustrate most of the network interactions taking place in real networks. We have assumed that generators are located at nodes 1 and 2 and that a consumption centre is connected to the transmission network at node 3 .

We assume that transactions carried out on the market led to the (net) schedules of (net) injections shown in Table 4.1, and Figure 4.1 shows the setting of our example. Note that positive net injections offset the negative net injections, so that the total schedules over the network are

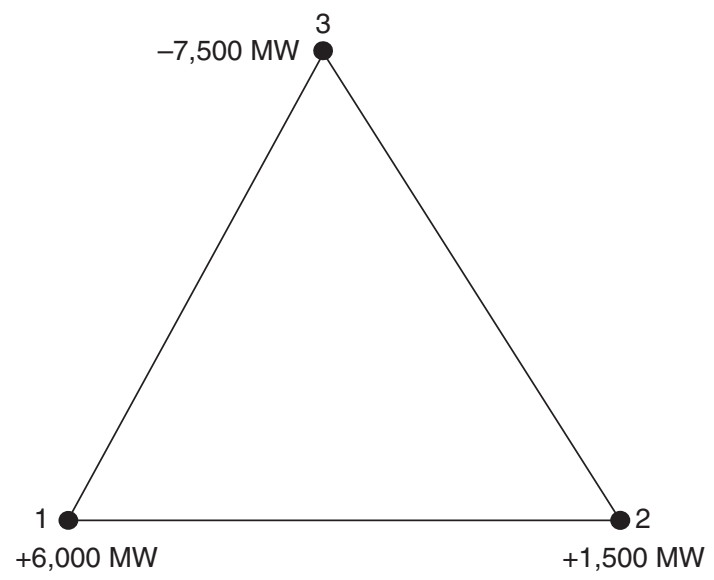

Figure 4.1 Triangular network example 
balanced. Although we have assumed for reasons of simplicity that only generators are connected at nodes 1 and 2 and only loads are connected at node 3 , this representation is general. Net injections at a node are the difference between total generation and total consumption taking place at the node. Therefore, a positive net injection indicates that generation at the node exceeds consumption, while a negative net injection indicates that consumption exceeds generation.

The set of net injections can be broken down into pairs of balanced injections and withdrawals, such that at each node the sum of net injections across all the pairs equals the total net injection. Thus, based on the superposition principle, power flows on the network will be determined as the sum of flows that would be independently caused by pairs of balanced net injections.

An example of the breakdown into balanced pairs of net injections is:

- a positive net injection of $6,000 \mathrm{MW}$ at node 1 , matched by a negative net injection of 6,000 MW at node 3; and

- a positive net injection of 1,500 MW at node 2, matched by a negative net injection of 1,500 MW at node 3 .

The inverse resistance rule allows a simple computation of the flows resulting from each pair of balanced net injections. For example, in the first pair the injection at node 1 is balanced by a withdrawal at node 3 . There are two paths between these two nodes: the short path $1 \rightarrow 3$, consisting of line $1-3$ and the long path $1 \rightarrow 2 \rightarrow 3$, consisting of lines 1-2 and 2-3. Since all lines are assumed to be identi$\mathrm{cal}$, the resistance of each path depends only on its length, and thus the resistance of the short path is half the resistance of the long path. The inverse resistance rule tells us that the flow produced by a transaction involving injections and withdrawals, respectively, at nodes 1 and 3 will split in the proportion of $2: 1$ between the short path and the long path. The flow on line $1-3$ of the short path will be $2 / 3 * 6,000=4,000 \mathrm{MW}$, and the flow over each of the lines $1-2$ and $2-3$ that make up the long path will be $1 / 3 * 6,000=2,000 \mathrm{MW}$. This is illustrated in the left panel of Figure 4.2.

Applying the same logic, the second injection at node 2 balanced with the withdrawal at node 3 results in a flow of $2 / 3 * 1,500=1,000 \mathrm{MW}$ over line $2-3$ and a flow of $1 / 3 * 1,500=500 \mathrm{MW}$ over each of the lines $2-1$ and $1-3$ that make up the long path between nodes 2 and 3 . This is illustrated in the second panel of Figure 4.3. Note that the direction of flows over each line is shown by the sign relative to the direction of the 

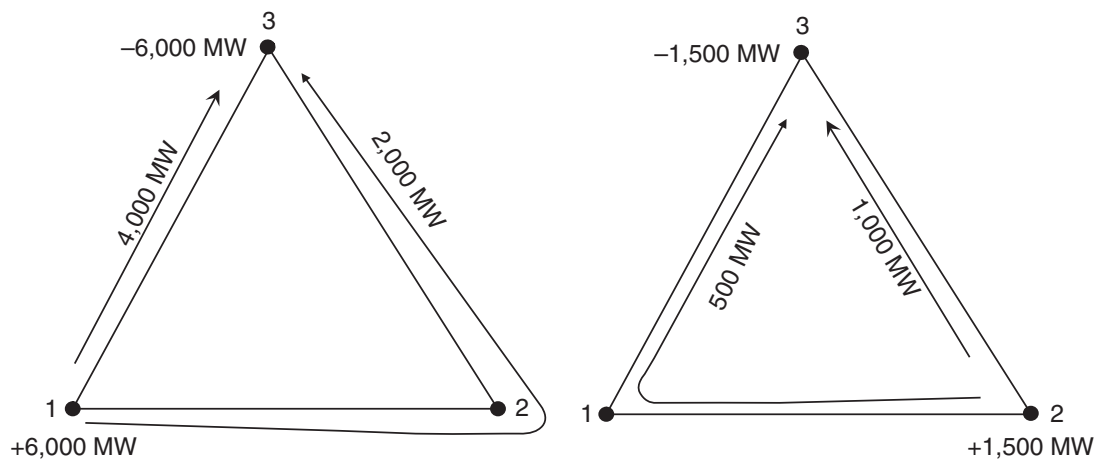

Figure 4.2 Power flows induced by injection-withdrawal pairs on a triangular network

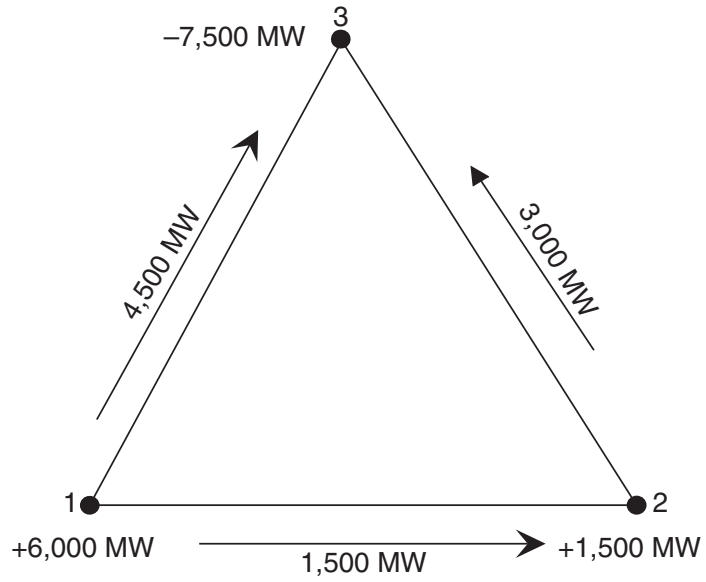

Figure 4.3 Superposition of the power flows

line: the flow of $500 \mathrm{MW}$ over line 2-1 is equivalent to the $-500 \mathrm{MW}$ flow over line 1-2.

The superposition principle tells us that the total flows over each line of the network are the sum of the flows independently determined for each pair of balanced net injections, taken with the correct sign. The result is shown in Figure 4.3.

The principle of superposition in the DC approximation makes it simple to calculate the flows brought about by a set of net injections even for large networks, reducing them to a set of linear equations. This calculation first requires a systematic way to break down the initial set of net injections 
Table 4.2 Net injections relative to the reference node $3(\mathrm{MW})$

\begin{tabular}{lc}
\hline Node & Net injection \\
\hline 1 & 6,000 \\
2 & 1,500 \\
\hline
\end{tabular}

into simple pairs of injections and withdrawals. This is done by selecting one of the network nodes as a reference or a swing node and assuming net injections at all other nodes to be balanced against that reference node. The initial set of net injections is then presented as a collection of independent net injections at all the nodes, each balanced against the reference node.

In the example above the two pairs of injections and withdrawals that we have considered were balanced at node 3 , which means that we have chosen node 3 as the reference or 'swing' node. The choice of swing node is arbitrary and does not change the result of the computation. What is important is that the choice remains consistent throughout the calculation. The net injections relative to the reference node used in our example are shown in Table 4.2.

The power injected at each node and withdrawn at the reference node is split across the lines making up the parallel paths as indicated in the power transfer distribution factor (PTDF) matrix. The PTDFs are defined in relation to the reference node and depend on the technical features and topology of the network. The PTDF matrix used in our example relative to reference node 3 is shown in Table 4.3. Note that the positive or negative sign of PTDFs determines the direction of flows with respect to the line. The negative PTDF of $1 / 3$ of node 2 relative to line 1-2 means that the net injection of $1 \mathrm{MWh}$ at node 2 withdrawn from the reference node 3 generates a flow of 1/3 MWh over line 1-2 from node 2 towards node 1 ,

Once the reference node has been set and the PTDF matrix for the reference node has been computed, the DC power flows over all the transmission elements are calculated using a set of linear equations based on the

\section{Table 4.3 PTDFs Matrix}

\begin{tabular}{lcc}
\hline Line & Node 1 & Node 2 \\
\hline $1-2$ & $1 / 3$ & $-1 / 3$ \\
$2-3$ & $1 / 3$ & $2 / 3$ \\
$1-3$ & $2 / 3$ & $1 / 3$ \\
\hline
\end{tabular}


net injections in all the remaining nodes. Power flow $F_{j}$ over transmission element $j$ is determined by:

$$
F_{j}=\sum_{i} P T D F_{i j}^{r} \cdot I_{i},
$$

where, $I_{i}$ is net injections at the node $i$ balanced at the reference node $r$, and $P T D F_{i j}^{r}$ is the PTDF of the net injection at node $i$ with respect to transmission element $j$, relative to reference node $r$. That is, the flow over each transmission element is the sum of net injections at all nodes of the network weighted by the flow sensitivities of this element to each node, given by the PTDF matrix.

\subsubsection{Congestion and Locational Differentiation of Electricity's Incremental Cost}

\section{Network constraints}

Elements of the transmission network may have certain limits on the power flow they can take. The simplest example is an overhead line that may overheat and stretch if the power flow is too large, with a risk of a short circuit. Transmission system operators often commit to maintain an $N-1$ reliability standard. This implies that the system should withstand an outage of any single transmission element at any time.

The $N-1$ standard results in contingency constraints. A contingency constraint limits the power flow over one line if another line, or network element, goes out of service. Since the outage may change the network topology, the contingency constraint may have different PTDFs compared with the all-in-service constraint on the same line. Regardless of the type, all constraints can be characterised by their PTDFs and the flow limit. Therefore, in the discussion that follows we do not distinguish between the types of constraint.

\section{Security domain}

Let us assume that in the setting introduced in the previous section, line 1-3 has a flow limit of 4,000 MW. In this case, the set of net injections assumed in the previous section becomes infeasible, since it induces a flow of 4,500 MW on line $1-3$, beyond the line's capacity. ${ }^{3}$ This is a congestion situation: generators and consumers wish to make injections and withdrawals producing flows that violate one or more network security constraints. In order to remain feasible, the net injections at nodes 1 and 2 have to be such that the power flows they create over line 1-3 remain within the line flow limits of 4,000 MW. Given the PTDFs of the net 


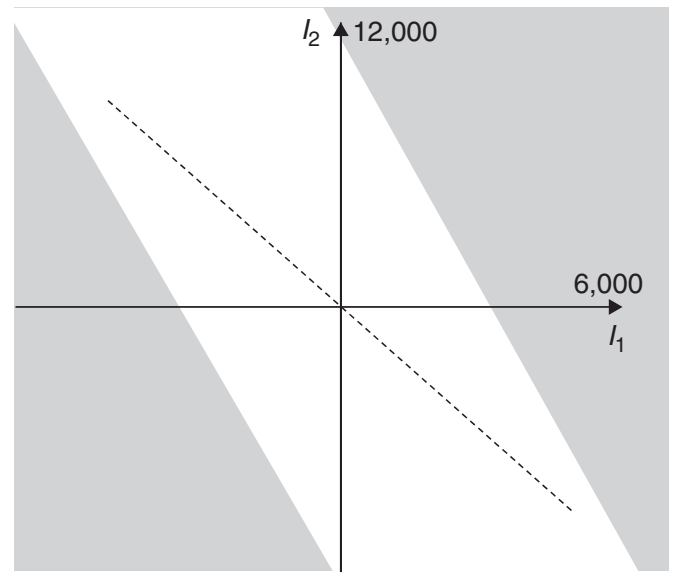

Figure 4.4 Security domain defined by the constraint on line 1-3

injections at nodes 1 and 2 with respect to this line of $2 / 3$ and $1 / 3$, respectively, this constraint can be written as:

$$
\frac{2}{3} I_{1}+\frac{1}{3} I_{2} \leq 4,000
$$

and the same constraint limiting the flow to 4,000 MW in the opposite direction is:

$$
-\frac{2}{3} I_{1}-\frac{1}{3} I_{2} \leq 4,000
$$

These constraints identify the set of all possible combinations of injections and withdrawals that are feasible, that is, that produce flows that do not violate any constraint. We refer to this set of feasible injections and withdrawals as the 'security domain'. In the case of a network with three nodes, the security domain can be shown on a two-dimensional graph, for example in terms of net injections at nodes 1 and 2, assuming that all such net injections are balanced at node 3 . The security domain defined by the constraint of 4,000 MW on line 1-3 is shown in Figure 4.4.

The security domain consists of the set of inequalities, each one limiting the flow along each line, expressed as a linear function of the injections at all nodes:

$$
F_{j}=\sum_{i} P T D F_{i j}^{r} \cdot I_{i} \leq F L_{j}
$$


Here, $F_{j}$ is the flow over transmission element $j, I_{i}$ is net injections at the node $i$ balanced at the reference node $r$, and $P T D F_{i j}^{r}$ is the PTDF of the net injection at node $i$ with respect to transmission element $j$, relative to reference node $r$, and $F L_{j}$ is the flow limit on constraint $j$.

\section{Locational incremental cost}

In the event of congestion the value of energy, that is, the minimum cost of meeting an incremental demand, differs at different nodes. Because of the network constraint, an additional withdrawal at a given node cannot necessarily be matched by increasing injections at the node where the cheapest available generation capacity is located. Instead, the lowest-cost option to match the additional demand at different nodes entails varying the injections of generators located at different nodes, and therefore results in additional costs.

In the setting introduced in the previous sections, let us assume that the incremental generation costs at nodes 1 and 3 are $€ 20 / \mathrm{MWh}$ and $€ 50 / \mathrm{MWh}$, respectively, and that there is unlimited production capacity at both these nodes. At node 2 the generation cost is $€ 10 / \mathrm{MWh}$ for production levels up to $1,500 \mathrm{MW}$, and very high for production levels above 1,500 MW. Note that these assumptions are consistent with the unconstrained market outcome assumed in the previous section: without network constraints the cheapest 7,500 MW generators in the system (1,500 MW at node 2 at $€ 10 / \mathrm{MWh}$ and 6,000 MW at node 1 at $€ 20 / \mathrm{MWh}$ ) would sell their production on the market.

With the network constraint on line 1-3, the demand for 7,500 MW at node 3 is met at minimum cost in the following way. First, 1,500 MW is injected at node 2, as this node has the cheapest power. However, the next cheapest node, node 1 , cannot meet the remaining 6,000 MW because the injection of 6,000 MW would breach the constraint on line $1-3$. The remaining demand is therefore met by 5,250 $\mathrm{MW}$ injected at node 1 and $750 \mathrm{MW}$ produced at node 3 , giving a net injection at node 3 of $-6,750 \mathrm{MW}$, in other words, a net withdrawal of 6,750 MW. This leastcost combination of net injections at nodes 1, 2 and 3 which respect the security constraint is shown in Figure 4.5.

We are now able to determine the lowest cost of matching an incremental demand increase at each node, that is, the value of energy at each node.

At node 1, an incremental withdrawal can be matched at minimum cost by increasing injections at the same node (production at node 2 is cheaper, but there is no spare capacity). Matching an incremental withdrawal with an injection at the same node does not change the net injection at the node, and therefore there is no impact on flows over the network. The 


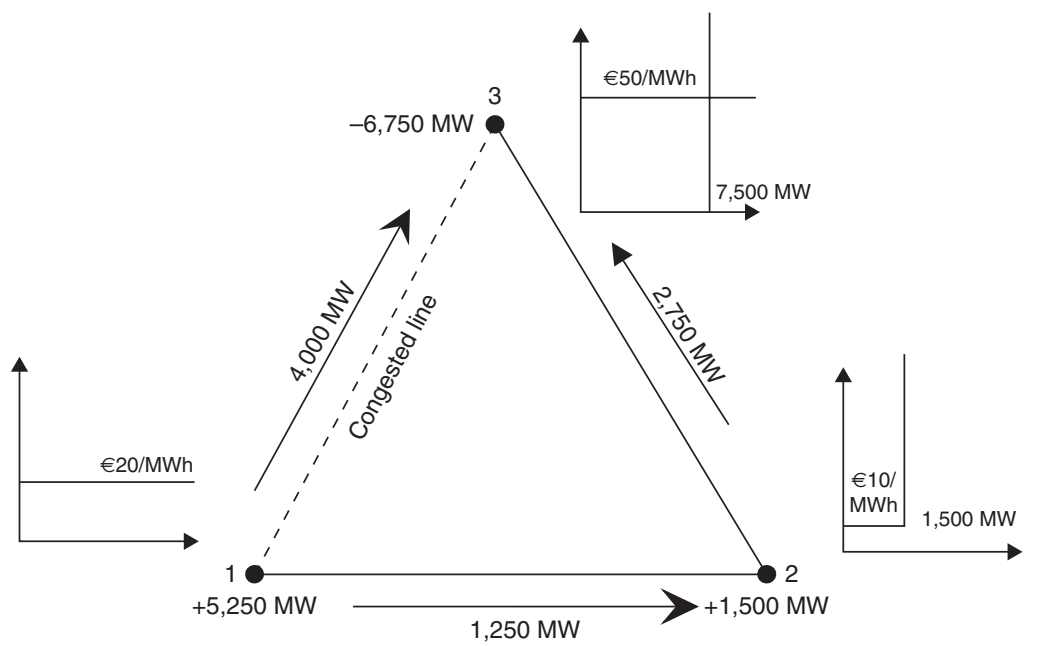

Figure 4.5 Power flows of the triangular network with congestion

incremental cost of withdrawals at node 1 is therefore $€ 20 / \mathrm{MWh}$, the marginal generation cost at node 1 .

At node 3, increasing injections at the same node is also the least-cost way to meet an incremental load at this node. Although production at nodes 1 and 2 is cheaper, they cannot be used to meet additional demand at node 3 . Additional production at node 1 would violate constraint $1-3$ and at node 2 there is no spare capacity. The incremental cost of withdrawals at node 1 is therefore $€ 50 / \mathrm{MWh}$, the marginal generation cost at node 3 .

The incremental cost of energy at nodes 1 and 3 is determined by the cost of the marginal units located at these nodes.

However, this does not hold for node 2 , because of the physics of power flows. The cheap production capacity at this node is fully utilised to meet demand. Thus, additional demand at node 2 must be met by production at other nodes, such as 1 and 3. The cheapest way to match such incremental load without violating the constraint on line $1-3$ is by increasing injections at nodes 1 and 3 by $1 / 2 \mathrm{MW}$ each. In order to understand that this is the least-cost combination of injections matching the incremental withdrawal at node 1 , consider that:

- the cost of this solution is $1 / 2 * 20+1 / 2 * 50=€ 35 / \mathrm{MWh}$;

- this solution creates zero incremental flow over the constrained line 1-3; and 
- any other feasible combination of net injections at nodes 1 and 3 would require a larger share of production to take place at the more expensive node 3 .

Thus, the incremental cost at node 2 is $€ 35 / \mathrm{MWh}$. This is determined by the cost of the variations in injections from the marginal generating units at nodes 1 and 3, so that these variations together produce zero incremental flow over the constrained line $1-3$ and the total of these variations is $1 \mathrm{MW}$.

In the above example, the constraint on line 1-3 has restricted the power flows between nodes 1 and 3 . As a result, the incremental costs of matching load in these nodes were set separately by marginal units at each of these nodes. Similar situations could arise if the power flows between nodes 1 and 3 were limited by constraints on lines $1-2$ or 2-3. However, each of these situations would imply a different marginal cost of energy at node 2 . Below we consider these two extensions of the previous example.

\section{Extension 1: constraint on line 1-2}

In this case we have assumed that line 1-2 has a flow limit of 1,000 MW, while there are no flow limits on other lines. This suggests that the minimum-cost injections and withdrawals shown in Figure 4.3 are not feasible. As in the main example, the cheapest capacity at node 2 is fully utilised and provides 1,500 MW. The remaining 6,000 MW of demand at node 3 is met by the generators at nodes 1 and 3 . The least-cost way of doing this without violating the constraint on line 1-2 is to produce 4,500 MW at node 1 and 1,500 MW at node 3 . This least-cost combination of net injections at nodes 1,2 and 3 , which respects the security constraint on line 1-2, is shown in Figure 4.6. Similarly to the main example, the incremental cost of energy at nodes 1 and 3 is determined by the cost of marginal units located at these nodes, that is, $€ 20 / \mathrm{MWh}$ at node 1 and $€ 50 /$ MWh at node 3 .

We shall focus on the incremental energy cost at node 2 . This cost is determined by the least-cost variation of injections at nodes 1 and 3 that together make up $1 \mathrm{MW}$ without changing the flow on the constrained line. A $1 \mathrm{MW}$ injection variation at node 1 would have a $2 / 3 \mathrm{MW}$ impact on the congested line, and a $1 \mathrm{MW}$ variation at node 3 would have $1 / 3 \mathrm{MW}$ impact on the congested line. Therefore, a double variation that would not violate the constraint would be a $2 \mathrm{MW}$ increase to the net injection at node 3 and a $1 \mathrm{MW}$ decrease in the net injection at node 1 . The cost of such a variation would be $2 * 50-1 * 20=€ 80 / \mathrm{MWh}$.

The marginal cost of energy at node 2 is higher than the cost of the marginal units at node 1 and node 3 . This is because additional demand 


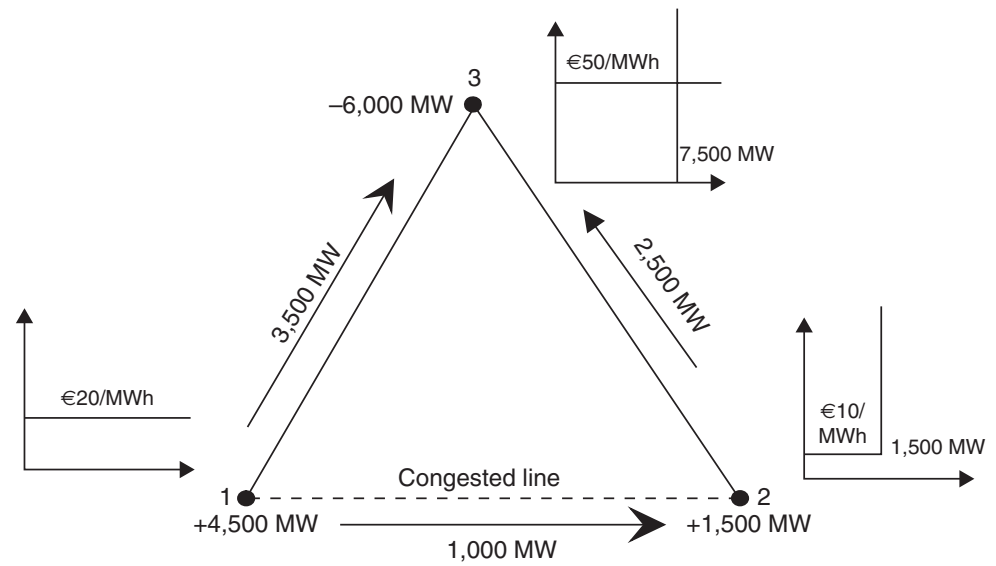

Figure 4.6 Power flows of the triangular network with congestion: constraint on line 1-2

at node 2 increases congestion. To meet the demand without violating the constraint, a costly counter-flow action would need to be implemented between the other two nodes. Thus, the energy cost at this node reflects the impact of this node on congestion in the rest of the network.

\section{Extension 2: constraint on line 2-3}

In this extension we have assumed that line 2-3 has a flow limit of 2,000 MW, while there are no flow limits on other lines. Again, this makes the minimum-cost injections and withdrawals shown in Figure 4.3 infeasible. Contrary to the previous examples, it is no longer optimal to use the cheapest capacity at node 2 . This is because when node 2 is used together with node 1 to meet the demand at node 3 , line $2-3$ very quickly becomes congested and a large share of the load at node 3 needs to be met by the expensive generators at that same node. It is cheaper not to use the capacity at node 2 at all, and to meet a larger share of the demand from the generators at node 1 . The resulting least-cost combination of net injections which respect the security constraint on line 2-3 is shown in Figure 4.7. As in the previous examples, the incremental cost of energy at nodes 1 and 3 is determined by the cost of marginal units located at these nodes, that is, $€ 20 / \mathrm{MWh}$ at node 1 and $€ 50 / \mathrm{MWh}$ at node 3 .

There is spare cheap generating capacity at node 2 at a cost of $€ 10 /$ MWh. However, it is not this capacity that determines the incremental energy cost at this node. Rather, as before, the energy cost at node 2 is determined by the least-cost feasible variation of injections at nodes 1 and 3 . This variation is a $2 \mathrm{MW}$ increase of the net injection at node 1 


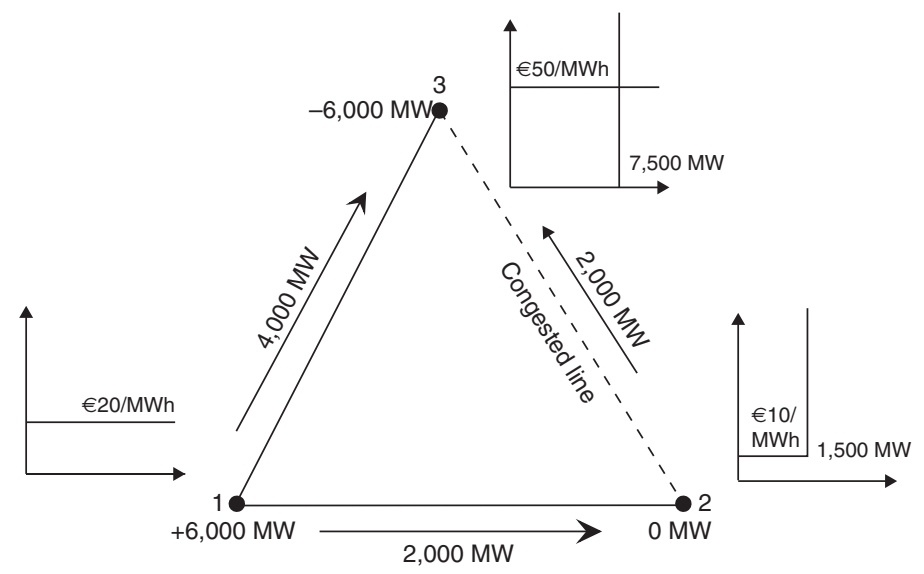

Figure 4.7 Power flows of the triangular network with congestion: constraint on line 2-3

and a $1 \mathrm{MW}$ decrease of the net injection at node 3 . The cost of such a variation would be $2 * 20-1 * 50=-€ 10 / \mathrm{MWh}$.

The marginal cost of energy at node 2 is negative. This is because additional demand at node 2 relieves congestion on the network. Meeting incremental demand at this node provides savings on the cost of the counter-flow action between the other two nodes.

\section{General formulation of nodal incremental costs}

In general, incremental energy costs at every node of the network are determined by an optimisation programme that minimises the cost of meeting demand in all locations by finding a set of generator injections at all network nodes, while ensuring that the flow constraints over all network elements are satisfied, in other words that the injections remain within the security domain. This optimisation programme is called 'security-constrained optimal dispatch'. A general outline of the optimisation programme can be written as:

$$
\min _{I_{i}} \sum_{i} C_{i}\left(I_{i}\right)
$$

The programme chooses the net injections at each node $i$ that will minimise their total generation cost. Alternatively, it can be presented as a choice of net injections that maximise the total benefit:

$$
\max _{I_{i}} \sum_{i} B_{i}\left(I_{i}\right) .
$$


Optimisation needs to ensure that the sum of the net injections over the entire system is zero. The optimisation therefore requires a balance constraint:

$$
\sum_{i} I_{i}=0
$$

Finally, optimisation requires a network constraint, in order to ensure that the chosen set of net injections remains within the security domain, or that the net flow over each line $j$ stays below the line's capacity:

$$
\sum_{i \neq r} P T D F_{i j}^{r} \cdot I_{i} \leq F L_{j}
$$

Incremental energy costs at all nodes are a byproduct of such dispatch optimisation. In the above examples the security-constrained optimal dispatch problems were simple enough to be solved manually and for the prices to be found. In large networks, security-constrained optimal dispatch becomes a complex optimisation problem. However, prices generated by these problems are relatively easy to analyse and to interpret thanks to several high-level properties. The following are some examples of these properties:

- Security-constrained economic dispatch determines the marginal cost of all flow constraints, sometimes called 'constraint shadow prices'. These represent the decrease in the total cost of meeting demand associated with a $1 \mathrm{MW}$ increase in the constraint limit. The constraint shadow price is only non-zero if the constraint is binding in the constrained economic dispatch solution.

- At each node the difference between the incremental energy cost and the reference node energy cost is given as the sum of the constraint shadow prices weighted by the relevant PTDFs.

- In the security-constrained economic dispatch solution the number of marginal units is at least equivalent to the number of binding constraints plus one. The marginal units determine the incremental energy cost at their respective nodes. Prices at the remaining nodes are related to the cost of the marginal units via PTDFs.

In real networks with hundreds of nodes, no more than a dozen of constraints are usually binding at one time. Thus the majority of the nodal incremental costs are not independently set by the cost of the marginal units located at the respective nodes, but are interrelated via the PTDFs and shadow prices. This relationship is as follows: 


$$
P_{i}=P_{r}-\sum_{j} P T D F_{i j}^{r} \cdot S P_{j}
$$

In this formula, $P_{i}$ is the incremental energy cost at node $i, P_{r}$ is the incremental energy cost at the reference node, and $P T D F_{i j}^{r}$ is the PTDF of the net injection at node $i$ with respect to transmission element $j$, relative to reference node $r$. Finally, $S P_{j}$ is the shadow price of constraint $j$.

\subsection{CONGESTION MANAGEMENT WITH NODAL PRICE DIFFERENTIATION}

Congestion management is a collection of market (and sometimes nonmarket) arrangements that ensure that power flows produced by power injections and withdrawals do not violate the constraints, that is, that power injections lie within the security domain.

In this section we discuss congestion management methodologies that allow market participants to conclude only market transactions resulting in feasible power flows. This is achieved by allocating and enforcing a set of rights to inject and withdraw power at different network locations.

These rights to use the transmission resources on the electricity spot market can be defined and allocated in different ways. In Sections 4.3.1 and 4.3.2 we analyse methodologies in which the set of rights made available to the market reflects each and every network constraint. In one case these rights are defined explicitly as the rights to cause a power flow over individual critical elements of the transmission networks, or flowgates. In the other case the rights are defined implicitly by means of centralised clearing of the market at different electricity market prices at different nodes. In Section 4.3.3 we discuss long-term financial transmission rights that are used in systems where congestion on the spot market is dealt with using centralised nodal markets with locational prices.

In this section we consider locations to be individual network nodes, but in Section 4.5 below we discuss methodologies in which similar explicit or implicit arrangements are defined over a simplified representation of the network that groups multiple nodes into large zones.

\subsubsection{Explicit Nodal Market: Flowgate Methodology}

We have begun our analysis using the flowgate approach because it is based on a highly intuitive definition of transmission rights. In the flowgate approach, transmission rights are defined as the rights to induce a power flow through a given network element, or flowgate. This implies that a party willing to complete a transaction entailing power injection at 


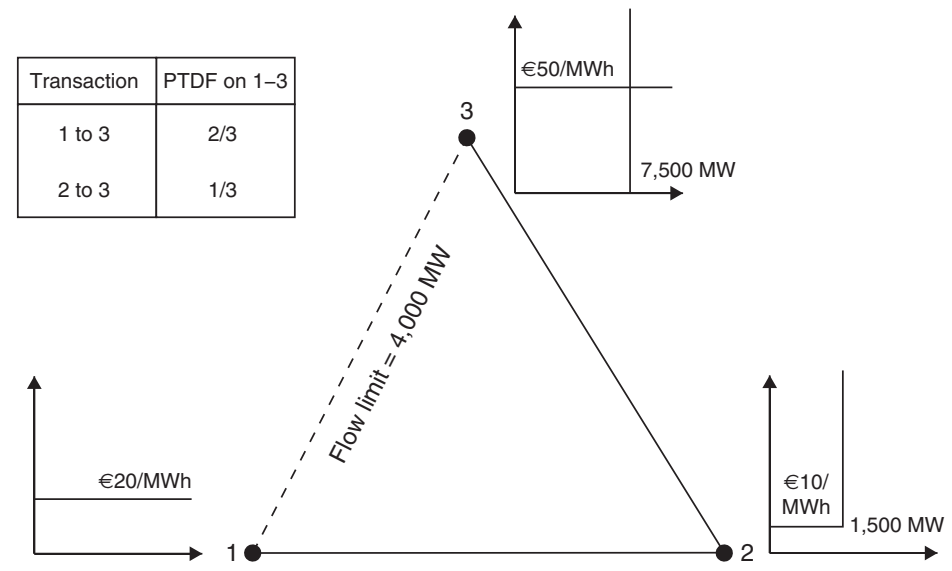

Figure 4.8 Triangular network with congested line and flowgate coefficients

a certain node and withdrawal at another node needs to procure the rights to use all network elements equivalent to the amount of the power flows over these elements ${ }^{4}$ produced by the transaction.

For each network element the system operator issues a number of flowgate rights equivalent to the network element flow limit. ${ }^{5}$ The system operator publishes the PTDFs of each flowgate relative to injections at each node matched with a withdrawal from the reference or swing node. These PTDFs can then be used to assess coefficients of flowgate utilisation by transactions involving any node pair.

We have used the simple setting developed in the previous sections to describe the market outcome in a system with flowgate rights. Figure 4.8 illustrates the example, which is consistent with those presented in Section 4.2.2 above. In this example the only network element with a flow limit is the line 1-3. The transmission system operator (TSO) issues the flowgate rights for this line for its flow limit of 4,000 MW and reports the PTDF coefficients representing usage of this flowgate by each transaction, specifically for the transactions from nodes 1 and 2 to node 3 .

We have assumed that the generators at nodes 1,2 and 3 carry out competitive bilateral trades to serve the demand of 7,500 $\mathrm{MW}$ at node 3. The value of the competitive bilateral deals over the network creates demand for use of the flowgate 1-3. The market price of these flowgate rights is determined by market participants' willingness to pay for the marginal MW of flowgate capacity.

For example, a generator at node 2 with a cost of $€ 10 / \mathrm{MWh}$ is willing 


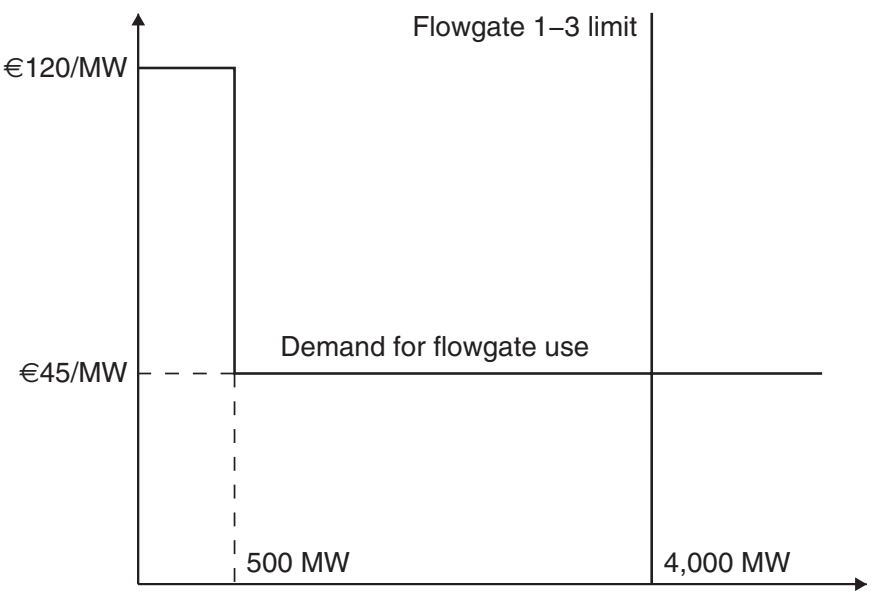

Figure 4.9 Demand for flowgate use

to pay up to $€ 40 / \mathrm{MWh}$ for the opportunity to serve the load at node 3 . If moving its production from node 2 to node 3 were more expensive, that generator would become uncompetitive compared with local production at node 3 , which has a cost of $€ 50 / \mathrm{MWh}$ and does not require flowing power through the flowgate. The PTDF matrix tells us that $1 \mathrm{MW}$ injected at node 2 and withdrawn at node 3 generates a 1/3 MW flow through the flowgate. Therefore, the willingness to pay for $1 \mathrm{MW}$ of the flowgate 1-3 by the generator at node 2 is $40 /(1 / 3)=€ 120 / \mathrm{MW}$. At that price, the entire available generating capacity of $1,500 \mathrm{MW}$ located at node 2 would be activated to serve load at node 3, after purchasing $500 \mathrm{MW}$ of the capacity of flowgate 1-3.

Likewise, a generator at node 1 with a cost of $€ 20 / \mathrm{MWh}$ is willing to pay up to $€ 30 / \mathrm{MWh}$ to serve load at node 3 . This is the difference between the cost of local supply at node 3 and the cost of the generator at node 1 . Since node 1 has $2 / 3$ sensitivity on the flowgate $1-3$, the generator is willing to pay up to $30 /(2 / 3)=€ 45 / \mathrm{MWh}$ for each MW of the capacity of the flowgate 1-3. The large amount of capacity available at node 1 means that the entire capacity of the flowgate is sold at the price of $€ 45 / \mathrm{MWh}$ to serve the load at node 3 .

Figure 4.9 illustrates the demand and the supply for the capacity of flowgate $1-3$. The clearing price of $€ 45 / \mathrm{MW}$ for flowgate $1-3$ is set at the intersection between the demand for flowgate use and the available flowgate capacity. The equilibrium of the flowgate and of the energy markets is such that the generator at node 2 produces 1,500 MW (impact of $500 \mathrm{MW}$ on the flowgate) and the generator at node 1 produces 5,250 MW (impact of 3,500 MW on the flowgate). The remaining demand is met by generation located at node 3 . 
Note that this competitive solution in the system with flowgate rights coincides with the least-cost dispatch presented in the baseline example in Section 4.2.2. The competitive price of $€ 45 / \mathrm{MW}$ of the flowgate constraint coincides with the shadow price of the constraint introduced in Section 4.2.2.

It is easy to verify that prices for electricity at each node can be calculated as the sum (or difference) of the marginal generation cost at the swing node (node 3 in our case) and the price of the flowgate right weighted by the sensitivity of the flowgate to the transaction between the given node and the swing node. In our example the price at the reference node 3 is set by the marginal cost of local generation of $€ 50 / \mathrm{MWh}$. The prices at nodes 1 and 2 are then calculated as follows:

$$
\begin{aligned}
& P_{1}=50-\frac{2}{3} 45=50-30=20 \\
& P_{2}=50-\frac{1}{3} 45=50-15=35 .
\end{aligned}
$$

More generally, the prices at all nodes in a market with flowgate rights are linked through the prices of all flowgate rights (SP) and node sensitivities with respect to those flowgates (PTDF):

$$
P_{i}=P_{r}-\sum_{j} P T D F_{i j}^{r} \cdot S P_{j} \cdot
$$

Our simple example shows that the market equilibrium with congestion features differentiation of the electricity prices by location, and that these prices are directly related to the prices of the flowgate transmission rights. The prices that clear the energy and the flowgate markets satisfy a non-arbitrage condition: the electricity price at each node is equal to the electricity price at any other node increased by the transmission cost between the two nodes.

In the event that a flowgate becomes congested its clearing price is positive. The total value of this flowgate right, the product of the flowgate price and the available rights through that flowgate, is commonly referred to as 'congestion rent'. In the example above this value is $4,000 \mathrm{MW}^{*} € 45 / \mathrm{MW}=€ 180,000$. We discuss congestion rents extensively in the following sections.

In the framework of decentralised negotiation assumed in this section, market clearing is achieved through the usual bilateral negotiation process between buyers and sellers of electricity and flowgate rights. In Chapter 2 we discussed extensively the rationale behind centralising electricity trades taking place near time of delivery. We argued that transaction costs make 
it unlikely that the decentralised trading mechanism results in an efficient outcome at all times. The argument becomes even more compelling in the case of congestion, when the market-clearing prices for electricity and flowgate rights need to be discovered simultaneously. For this reason the flowgate approach has rarely been implemented in real electricity markets. Perhaps the only example is the electricity market of Texas, where flowgate rights were implemented in 2002 until the reform of the market in 2009-10. ${ }^{6}$

\subsubsection{Implicit Nodal Market: Bid-based Security-constrained Economic Dispatch}

In the previous section we discussed the impact of transmission congestion on the market outcome in a bilateral trading framework that highlights the analogies between electricity and all other goods. In this section we discuss the alternative market design in which electricity trading is centralised, and the rights to use the transmission network are allocated implicitly as part of the clearing process of the electricity market. The model is a stylised version of the standard market design of the nodal electricity market implemented in most US wholesale markets.

In this congestion management methodology the electricity spot market is cleared through an auction, like the one discussed in Chapter 2.7 Generators and load-serving entities submit offers to sell and bid to buy electricity at the nodes where they are located. The clearing algorithm selects the set of offers and bids that:

- maximise the net surplus resulting from the transactions, and

- do not violate any network security constraints.

The market-clearing solution of security-constrained surplus maximisation produces a market-clearing price at each network node. This price is paid for all accepted sell offers and charged to all accepted buy bids at that node. In case of congestion the market-clearing prices at different nodes differ, to reflect the different cost of meeting the incremental demand at each location.

Flowgate rights are not explicitly allocated in the centralised setting; however, the set of transactions that market participants are allowed to carry out is limited to those meeting all the network-related constraints. For a given set of such constraints, with no transaction costs, the same market outcome would result from the centralised setting of a nodal market as from the decentralised setting with flowgate rights. In practice, however, because of transaction costs, the two implementations could yield different outcomes. 
The market operator collects the congestion rent, which is the difference between the revenues from accepted bids to buy and the payments for the accepted offers to sell. It is simple to show that the congestion rent collected in this setting is identical to the congestion rent obtained in the alternative setting from the sale of flowgate rights. The congestion rent collected on the nodal market is the product of the net injections at each node and the clearing nodal price:

$$
C R=-\sum_{i} P_{i} \cdot I_{i}=\sum_{i \neq r}\left(P_{r}-P_{i}\right) \cdot I_{i}
$$

Bearing in mind the relationship between the nodal prices and constraint shadow prices, this becomes:

$$
C R=\sum_{i \neq r}\left(\sum_{j} P T D F_{i j}^{r} \cdot S P_{j}\right) \cdot I_{i}=\sum_{j}\left(\sum_{i \neq r} P T D F_{i j}^{r} \cdot I_{i}\right) \cdot S P_{j} .
$$

If we use the relationship between the nodal net injections and flows over the transmission elements from the DC flow model, the congestion rent becomes:

$$
C R=\sum_{j} F_{j} \cdot S P_{j}
$$

We should also recall that the constraint shadow price is non-zero only in the case of the constraint being binding and the flow being equivalent to the flow limit. Thus, the congestion rent is also equivalent to:

$$
C R=\sum_{j} F L_{j} \cdot S P_{j}
$$

where $F L_{i}$ is the flow limit transmission element $j$. This dual representation of congestion rent through nodal prices and nodal net injections on the one hand, and flow limits and shadow prices on the other, proves very useful when there is a need to identify the congestion rent generated by each transmission element.

\subsubsection{Long-term Financial Transmission Rights}

The spot market cleared at a nodal level may produce substantial differences between energy prices at different locations and create significant volatility in the congestion charges. Efficient trading may require instruments that allow market participants to hedge locational price risks. Forward financial transmission rights (FTRs), are instruments used on locational-based markets for that purpose. 
An FTR with a contractual volume of $1 \mathrm{MW}$ between injection point A and withdrawal point $\mathrm{B}$ entitles its holder to receive the difference between the locational spot prices at points $\mathrm{B}$ and $\mathrm{A}$ over the period covered by the contract. Therefore, if the volume of FTRs between two nodes matches the traded volume between them, an FTR becomes a perfect hedge against the cost of congestion.

FTRs typically have a duration of between one month and several years, and provide economic signals for the location of generators and customers. We discuss below how FTRs can also be used to provide incentives for investment in transmission.

\section{Simultaneously feasible transmission rights and revenue adequacy}

We assume here that the system operator, which also acts as the market operator, issues the FTRs. By issuing the FTRs the system operator commits to pay its holders the differences in energy market prices between pairs of nodes. This commitment is simple to implement when the net nodal injections resulting from the market clearing coincide exactly with the set of allocated FTRs. In this case the total FTR payment will precisely match the congestion rent collected by the market operator as a result of market clearing.

However, more often this is not the case and market-clearing injections and withdrawals differ significantly from the allocated FTRs. Yet this does not mean that the debts of the system operator to the FTR holders will outweigh congestion rents, placing financial risk on the market operator. In fact, provided the FTRs are simultaneously feasible, irrespective of the market outcome, the total payout to the FTR holders cannot exceed the congestion rent collected by the market operator. This result is commonly known as 'revenue adequacy'.

A set of FTRs is simultaneously feasible if the physical injections and withdrawals perfectly matching the set would not produce flows breaching any constraints. In other words, the set of FTRs is simultaneously feasible if the network can carry under security conditions the flows produced by their simultaneous exercise. In order to ensure that the set of FTRs allocated to the market is simultaneously feasible, the market operator carries out a simultaneous feasibility test. This process involves running an optimisation programme in which the objective function of the FTR allocation mechanism (for example, the auction value of the allocated FTRs) is maximised under the constraint that the sum of allocated FTRs at each node would produce feasible flows. This constraint can be presented as:

$$
F_{j}^{F T R}=\sum_{i \neq r} P T D F_{i j}^{r} \cdot F T R_{i} \leq F L_{j}
$$


Simultaneously feasible FTRs

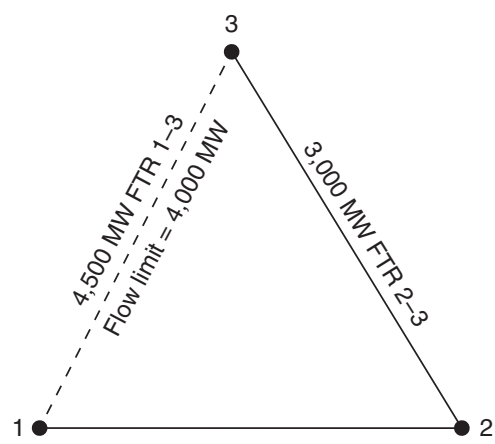

Market solution

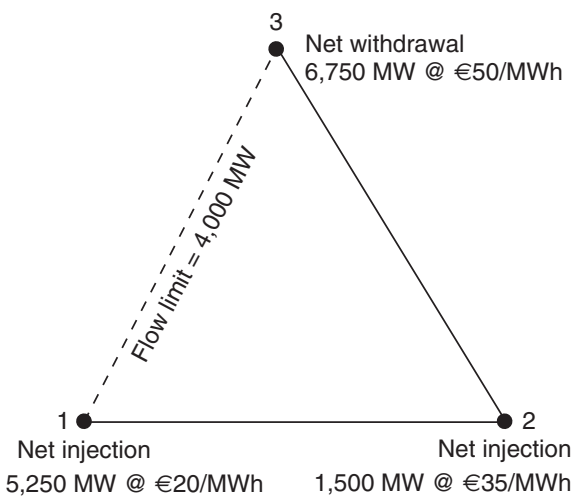

Figure 4.10 Simultaneously feasible FTRs

where $F_{j}^{F T R}$ is the flow that would be produced by net injections matching the set of FTRs over line $j$, and $F L_{j}$ is the flow limit of line $j$.

We shall illustrate revenue adequacy in Figure 4.10 using our usual example of a triangular network in which a single line 1-3 has a flow limit of 4,000 MW. We consider a set of FTRs comprising 4,500 MW FTRs between nodes 1 and 3 and 3,000 MW FTRs between nodes 2 and 3. It is simple to verify that this set of FTRs is feasible. The flow over line 1-3 produced by FTR $1-3$ is 3,000 MW (2/3 of 4,500 MW) and the flow over line 1-3 produced by FTR $2-3$ is $1,000 \mathrm{MW}(1 / 3 /$ of $3,000 \mathrm{MW})$. Obviously, this is not the only possible set of feasible FTRs. For example, other feasible sets could be:

- 6,750 MW FTR 1-3 and 1,500 MW FTR 2-1; and

- 7,500 MW FTR 1-3 and 3,000 MW FTR 3-2.

Now let us assume that the market outcome was that of the previous example in Figure 4.5, above, that is, that the net injections at nodes 1, 2 and 3 were 5,250 MW, 1,500 MW and -6,750 MW respectively, and prices were $€ 20 / \mathrm{MWh}, € 35 / \mathrm{MWh}$ and $€ 50 / \mathrm{MWh}$, respectively. We can calculate the congestion rent accrued by the market operator as the sum of withdrawals multiplied by nodal prices less the sum of injections multiplied by the nodal prices:

$$
C R=-5,250 \cdot 20-1,500 \cdot 35+6,750 \cdot 50=€ 180,000 .
$$


The payout due to the holders of allocated FTRs can be calculated by multiplying each FTR by the difference between corresponding nodal prices:

$$
\text { FTR payout }=(50-20) \cdot 4,500+(50-35) \cdot 3,000=€ 180,000 .
$$

This means that even though the FTRs do not initially match the market-clearing injections, the FTR payouts can be fully funded by the congestion rent collected on the market.

This is no coincidence. Readers can repeat this exercise for the other feasible FTR sets mentioned above. To illustrate this in a general sense we should recall that congestion rent can be expressed in terms of constraint shadow prices and the flow limits:

$$
C R=\sum_{j} F L_{j} \cdot S P_{j}
$$

The FTR payout, on the other hand, is:

$$
\text { FTR payout }=\sum_{i \neq r}\left(P_{r}-P_{i}\right) \cdot F T R_{i}=\sum_{j} F_{j}^{F T R} \cdot S P_{j},
$$

where $F_{j}^{F T R}$ are the flows produced by the set of FTRs on lines $j$. The revenue adequacy holds as long as the flows produced by the allocated FTRs over each line are within the limit, that is, as long as the FTR set is simultaneously feasible:

$$
\text { FTR payout }=\sum_{j} F_{j}^{F T R} \cdot S P_{j} \leq \sum_{j} F L_{j} \cdot S P_{j}=C R .
$$

We have shown that revenue adequacy holds in a simplified lossless DC approximation of transmission flow that we use throughout this book. However, revenue adequacy has been shown to hold in many flow models that are much closer to reality, such as a DC approximation with losses, or even an AC model. In fact revenue adequacy holds as long as the security domain defined by the network constraints is convex.

Most importantly, revenue adequacy holds as long as the network capacity remains the same from the moment the FTRs are allocated to the moment of market clearing. A change in network capacity could be, for example, a change in the flow limit of a constraint or the outage of a transmission element that could change both the PTDFs and some of the limits on the remaining transmission constraints. Even if the set of FTRs were feasible at the time of allocation, the changes in network topology could make this set infeasible on the network used for market clearing. Revenue inadequacy on a particular constraint will occur any time the constraint 
becomes binding in the market solution, and when the set of allocated FTRs induce a flow that exceeds the limit of this constraint.

Consider that in our example the flow limit of the line 1-3 was 4,000 MW when the simultaneous feasibility test was carried out, and therefore the awarded set of FTRs was feasible. Assume now that at the time of market clearing the limit decreased to 3,500 MW. The new limit does not change the market prices or constraint shadow price, but it does change the market-clearing volume of injections at nodes 1 and 3 .

Since the prices are the same as before, the payout due to the FTR holders would also be the same, $€ 180,000$. However, we can calculate the new congestion rent, which will be the product of the constraint shadow price of $€ 45 / \mathrm{MWh}$ and the new line limit of $3,500 \mathrm{MW}, € 157,500$. We find that the decrease of the limit on the 1-3 line causes a revenue shortfall of $€ 22,500$ for the market operator.

Since FTRs are purely financial instruments, nothing prevents any economic agent from issuing them. However, as the discussion on simultaneous feasibility has shown, only the party that collects the congestion rent is hedged against the obligations falling on the FTR issuer. Any other issuer would be taking a purely speculative position on the price differences across locations. For this reason the system operator is the main if not the only issuer of FTRs in most electricity markets.

\section{FTR allocation}

There are many ways to allocate FTRs for existing transmission capacity. In most cases FTRs are auctioned off by the system operator to market participants. In FTR auctions the system operator may also select the set of FTRs that has the highest value for market participants, while being simultaneously feasible. The outcome of the auction is the allocation of FTRs to the market players and the setting of market-clearing FTR prices.

The FTRs achieve liquidity on the secondary markets, where market participants can exchange and reconfigure their FTR positions following developments in market conditions.

The revenue collected by system operators in FTR auctions is another form of congestion rent; it is actually the long-term expectation of congestion rent. These revenues are typically passed on to the transmission service customers, in the form of a reduction in transmission tariffs.

In some countries FTRs have been grandfathered to market participants based on pre-existing allocation of explicit flowgate-like transmission rights, or as a way of limiting the economic impact on existing generators of the introduction of a congestion management system based on locational prices. 


\section{FTR obligations and options}

The discussion so far has assumed that FTRs are obligations, that is, the holder of an FTR is entitled to the difference between the market price at the withdrawal and injection point, regardless of whether the difference is negative or positive. If the price difference is negative, the holder pays the market operator the price difference.

In addition to these FTR obligations, market participants are often interested in the opportunity to hedge their position using FTR options. These options allow the FTR holder not to have to pay the market operator the price difference if it is negative.

An FTR option can be considered an FTR obligation that exists when the price difference between its withdrawal and injection points is positive, and disappears when the price difference turns negative. The feasibility of FTR options deserves special attention. A set of such FTRs should not only be feasible for injections and withdrawals matching all FTRs; it should also be feasible in case part of all the injections and withdrawals matching FTR options did not take place.

We can illustrate this in the context of the above example considering the following set of FTRs:

- A is an FTR obligation of 7,500 MW between nodes 1 and 3, and

- $\mathrm{B}$ is an FTR option of 3,000 MW between nodes 3 and 2.

The two FTRs are feasible if the price difference between nodes 3 and 2 is positive. The FTR A creates the flow over the constraint 1-3 of 5,000 MW, and the FTR B creates a counter-flow of 1,000 MW over this constraint. However, if the price difference is negative the flow matching FTR option $\mathrm{B}$ is not generated by the market equilibrium injections and withdrawals, making FTR A infeasible by itself.

The maximum number of FTR obligations between nodes 1 and 3 that can be allocated together with FTR option B is 6,000 MW. These FTRs will fully utilise network capacity in case the price difference between nodes 3 and 2 is negative so that FTR option B is not exercised. However, when the price difference is positive, the line capacity cannot be sold forward without placing financial risk on the market operator.

\subsection{CONGESTION MANAGEMENT VIA RE-DISPATCH}

Methodologies to deal with network constraints discussed in the previous section are based on restricting electricity market transactions to those 
that the network can safely accommodate. This is achieved by allocating to network users a feasible set of rights to inject and withdraw power at the different network locations. In the event of congestion, different electricity prices are set in different locations to enforce a feasible market outcome. These locational prices ensure that quantities that are economic to produce in each location induce flows over the network that do not violate transmission constraints.

In this section we discuss an alternative congestion management approach, consisting of two stages. In the first stage, market transactions are not subject to any network-related restrictions. Injections and withdrawals ensuing from this first stage may be infeasible, leading to flows that violate transmission constraints. This congestion is relieved in the subsequent stage, through re-dispatch, that is, by instructing and paying generators and possibly consumers to modify their injections and withdrawals at specific locations. In this section we analyse the features of the re-dispatch approach as well as certain bidding incentives that it creates. Later, in Section 4.6 we review the longstanding policy debate on the relative merits of congestion management via re-dispatch, nodal price differentiation or an intermediate zonal approach discussed in Section 4.5.

In Section 4.4.1 we introduce and illustrate the re-dispatch approach using a simple example. In Section 4.4.2 we present the bidding incentives created by this congestion management system and discuss some undesirable consequences of such bidding.

\subsubsection{Principles of Re-dispatch Congestion Management}

In the re-dispatch approach, market transactions taking place in the first stage are carried out assuming unlimited transmission capacity within national borders, and are not subject to any network-related restrictions. Market players buy and sell electricity, bilaterally or through power exchanges, knowing that the delivery and collection obligations corresponding to their sales and purchases can be fulfilled by, respectively, injecting or withdrawing power at any location of the network node of their choice. As a consequence, the energy market clears with a single system-wide price, as injections at any location are assumed to be perfect substitutes, as are withdrawals.

However, since no network-related restrictions are placed on the market players, power injections and withdrawals scheduled in the market equilibrium may violate network constraints. Congestion could be identified by the system operator based on generators' nominations (also known as 'schedules' or 'programmes') received after gate closure of the market.

Congestion resulting from the first unconstrained stage is relieved at a 
subsequent stage, through re-dispatch, that is, by paying generators and possibly consumers to reduce or increase injections and withdrawals at selected nodes.

The re-dispatch approach is widely adopted in Europe to relieve transmission congestion within national borders in such countries as the UK, France, the Netherlands, Spain and Germany. In part, the rationale for such market organisation is the perception that congestion within each national market is infrequent, and that little re-dispatch is required to make the unconstrained market solution feasible. We refer to such systems as re-dispatch or 'single-price' systems.

The second stage of re-dispatch congestion management is generally performed through a bidding market. This can be run immediately after the first-stage unconstrained market clearing, like in Spain, where the re-dispatch market (restrictions market) is run immediately after the unconstrained day-ahead single-price market. Alternatively, the re-dispatch market can be run closer to real time, as in the UK electricity market, where re-dispatch is performed as a part of the balancing mechanism.

Bids to reduce output collected on the re-dispatch market reflect the price that a generator is prepared to pay in order to scale back the production of a specific plant; conversely bids to increase output reflect the price that a generator agrees to receive in order to increase the production of a specific plant. In the event of congestion, the system operator selects the highest bids to reduce output compared with the day-ahead schedule from the generators located on the export side of the constraint, and the lowest bids to increase output from the generators located on the import side of the constraint. The aim of this bid selection is to modify the dispatch of plants in order to relieve congestion at the lowest cost while maintaining the energy balance within the control area.

Generators selected for schedule change during this process pay or are paid the value of their bids. Generators in the export area, whose bids to reduce output are accepted, buy energy back from the system operator at the bid price, instead of generating that energy themselves.

The price paid to the system operator by generators to reduce output in the export-constrained area is generally lower than the unconstrained market price. Conversely, the bid price to increase output paid by the TSO to generators in the import-constrained area is typically higher than the unconstrained market price. In this section we refer to the prices of accepted re-dispatch bids as 're-dispatch prices'.

As a result of re-dispatch, the system operator faces the costs of relieving congestion, since the cost of purchasing additional injections in the import-constrained area is greater than the revenues collected in the 


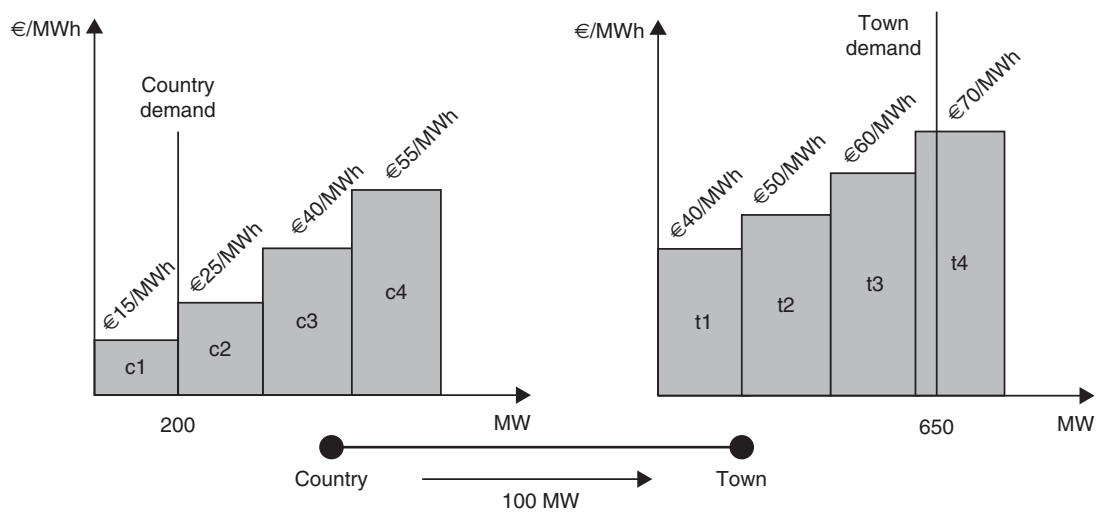

Figure 4.11 Example set-up

export-constrained area from the generators called upon to reduce injections. These costs are generally socialised among market participants.

\section{Example of re-dispatch congestion management}

We illustrate the re-dispatch approach using a simple example. The focus of this example is bidding and bidding incentives rather than the effects of power flows over meshed transmission networks as in previous examples. Therefore we consider an example with two areas only, connected by a transmission link: Country and Town. The Country area has four generators, each with $200 \mathrm{MW}$ of capacity. This area has consumption of $200 \mathrm{MW}$. The Town area also has four generators, each with $200 \mathrm{MW}$ of capacity, but it has a higher energy demand of $650 \mathrm{MW}$. Generators located in the Country have somewhat lower variable production costs than generators located in the Town. The capacity of the transmission line connecting the two areas is $100 \mathrm{MW}$. This example is illustrated in Figure 4.11.

Now we assume that the demand in this market has been cleared in the day-ahead market. This market will be the first stage of the re-dispatch congestion management, in which the transmission constraints impose no limits on market transactions. To make it simpler we shall also assume for now that market participants are not aware of the presence of the transmission constraint.

We assume (as is quite often the case) that the day-ahead market has a non-discriminatory auction design. In this market the clearing price is determined by the highest accepted bid, and all accepted supply bids are paid this clearing price. In a competitive environment this auction design is known to induce bidding by generators close to marginal cost of 


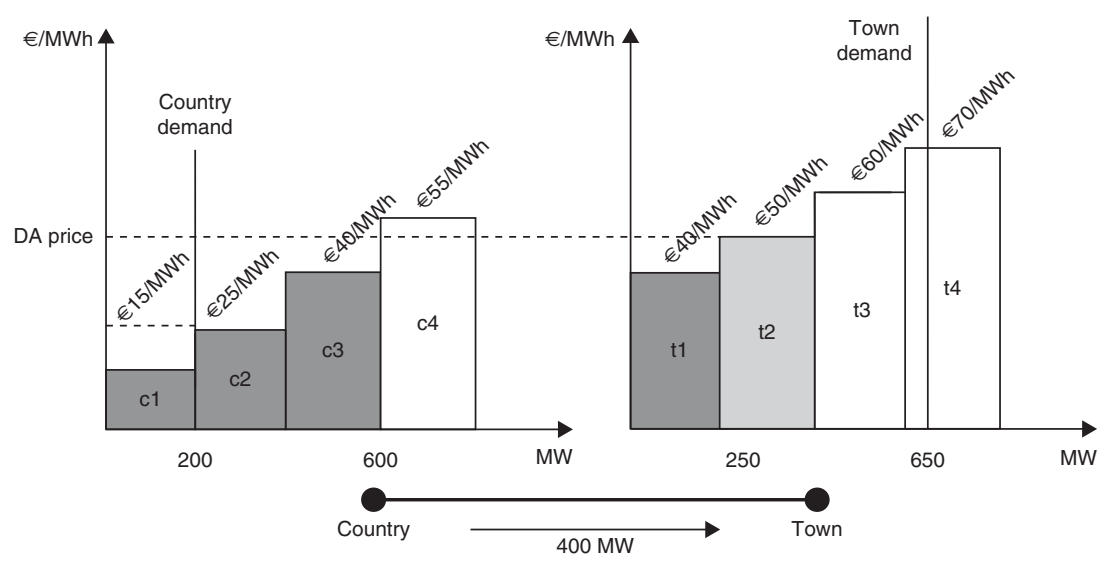

Figure 4.12 Unconstrained market outcome: no re-dispatch bidding incentives

production. The outcome of the day-ahead (DA) market, assuming such cost-based bidding takes place, is shown in Figure 4.12.

The least-cost way to meet the total demand of $850 \mathrm{MW}$ in both areas is to use the cheapest available units in both areas. This involves scheduling at full capacity the three cheapest units located in the Country area, c1 to c3, and one unit t1 from the Town area. Finally, unit t2 is scheduled to produce $50 \mathrm{MW}$ to meet the remaining $50 \mathrm{MW}$ of demand. Unit t2 is marginal in the day-ahead market and sets the day-ahead price at $€ 50 / \mathrm{MWh}$. This day-ahead market outcome produces a power flow of $400 \mathrm{MW}$ from the Country area to the Town area.

Upon clearing the day-ahead market the system operator realises that the power flow of $400 \mathrm{MW}$ induced by the day-ahead schedules violates the constraint of $100 \mathrm{MW}$ between the two areas, and re-dispatch is needed to reduce this flow by $300 \mathrm{MW}$. We consider first the cost-based merit order of units that are available to perform the necessary re-dispatch on both sides of the constraint. The least-cost way to eliminate the congestion involves reducing the output of the highest-cost scheduled units in the Country area by $300 \mathrm{MW}$. This requires reducing the entire output of $\mathrm{c} 3$ and $100 \mathrm{MW}$ of the output of $\mathrm{c} 2$. The least-cost way to eliminate the congestion involves increasing the output of the lowest-cost available units in the Town zone by $300 \mathrm{MW}$. That requires increasing the output of $\mathrm{t} 2$ by $150 \mathrm{MW}$ and increasing the output of $\mathrm{t} 3$ by $150 \mathrm{MW}$.

Unit $\mathrm{c} 2$ is the marginal unit running in the export-constrained Country area after this cost-based re-dispatch, and the variable cost of c2 (€25/MWh) represents the marginal cost of energy in this area. Unit 


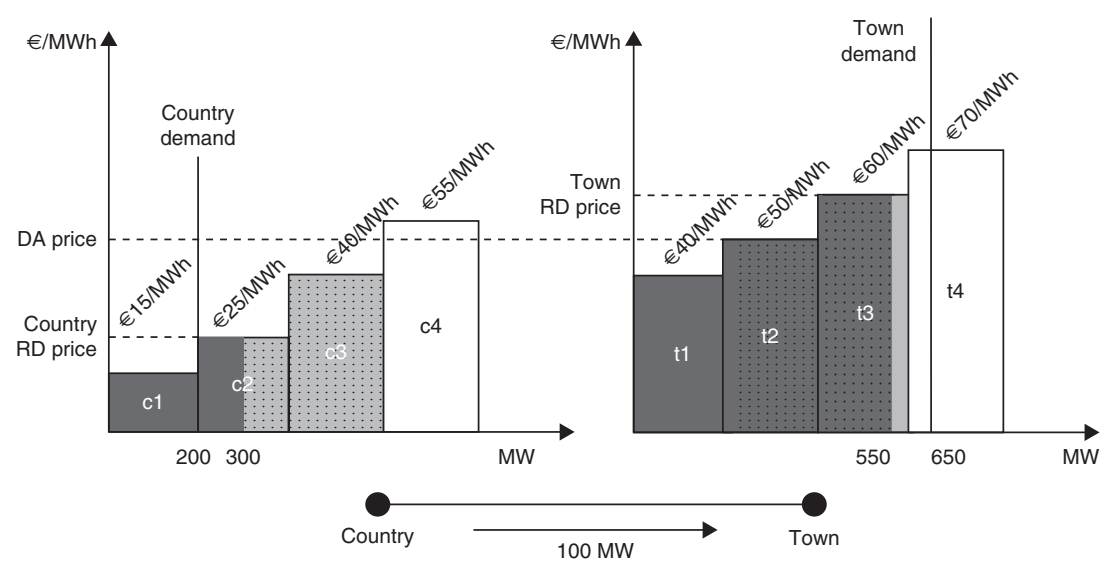

Figure 4.13 Cost-based re-dispatch and the equilibrium area prices

t3 becomes the marginal unit running in the import-constrained Town area in the cost-based re-dispatch, and its variable cost of $€ 60 / \mathrm{MWh}$ is the marginal cost of energy in this area after taking into account the constraint. In a competitive re-dispatch market, these marginal energy costs in each area would determine the competitive prices in each area. Such competitive prices would also be set in the case of locational market clearing.

Figure 4.13 illustrates the re-dispatch (RD) prices determined by the marginal cost of the marginal unit running in each area after cost-based redispatch, as well as the day-ahead price that would prevail in the absence of congestion.

\subsubsection{Bidding Incentives Created by Re-dispatch Congestion Management}

The re-dispatch market may have an important impact on the bidding behaviour of competitive generators: if market participants can predict that there will be congestion that will be resolved in the re-dispatch market, they will bid differently in the day-ahead market.

\section{Day-ahead bidding by competitive generators expecting a transmission constraint}

When we first considered the unconstrained day-ahead market in our example, we assumed that market participants would be unaware about the network constraint, and each competitive generator would bid according to its marginal cost. However, in a market where congestion management is carried out via re-dispatch, if congestion is predicted, rational 


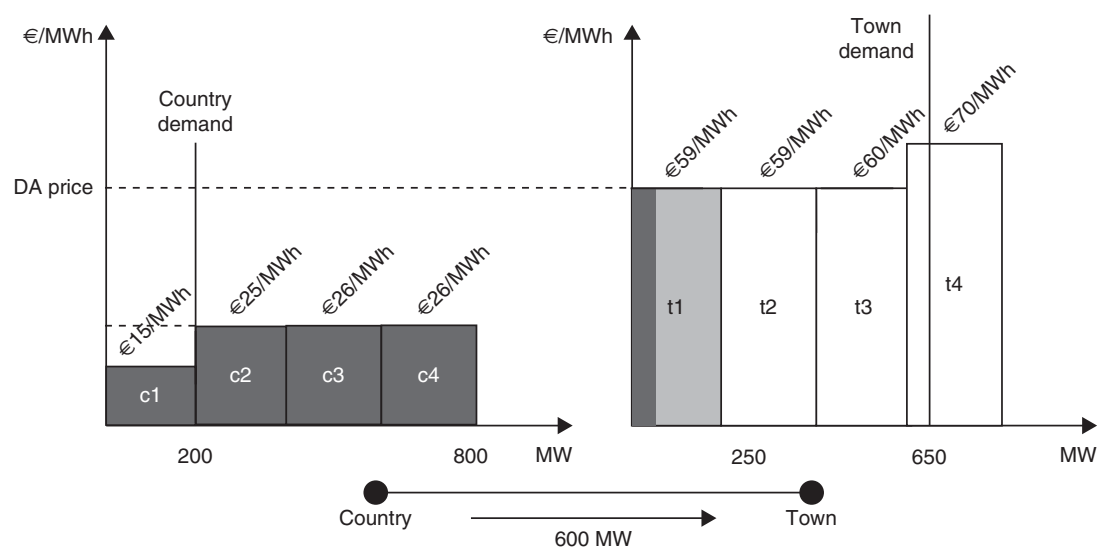

Figure 4.14 Illustration of the arbitrage between day-ahead and re-dispatch market

competitive generators will realise that submitting cost-based day-ahead bids as presented above is not profit maximising.

Generators would expect that, because of the transmission constraint, the re-dispatch price at their location would be different from the nationwide uniform day-ahead energy price, providing them with an opportunity to arbitrage between these two markets. In order to perform arbitrage between the day-ahead and the re-dispatch market, generators would submit bids in the day-ahead market departing from their variable production costs. Instead, their day-ahead bids would be driven by the opportunity costs determined by the price they could get in the re-dispatch market in their respective areas.

In our example, these bidding incentives mean that the entire capacity of unit $\mathrm{c} 4$ in the Country area and the entire capacity of unit $\mathrm{t} 1$ in the Town area will be bid for in the day-ahead market at opportunity cost. The opportunity costs are aligned with the competitive re-dispatch prices in their respective areas. The outcomes of the day-ahead and the re-dispatch markets are illustrated in Figures 4.14 and 4.15.

Figure 4.14 suggests that all generators in the Country with a variable cost above the re-dispatch price would submit day-ahead bids at that re-dispatch price. At the same time, all generators in the Town with a variable cost below the re-dispatch price would submit day-ahead bids at that re-dispatch price. The re-dispatch price in each area represents the opportunity cost of these units.

Such bidding changes the outcomes of the day-ahead market compared with the cost-based bidding presented above. In the first place, the 


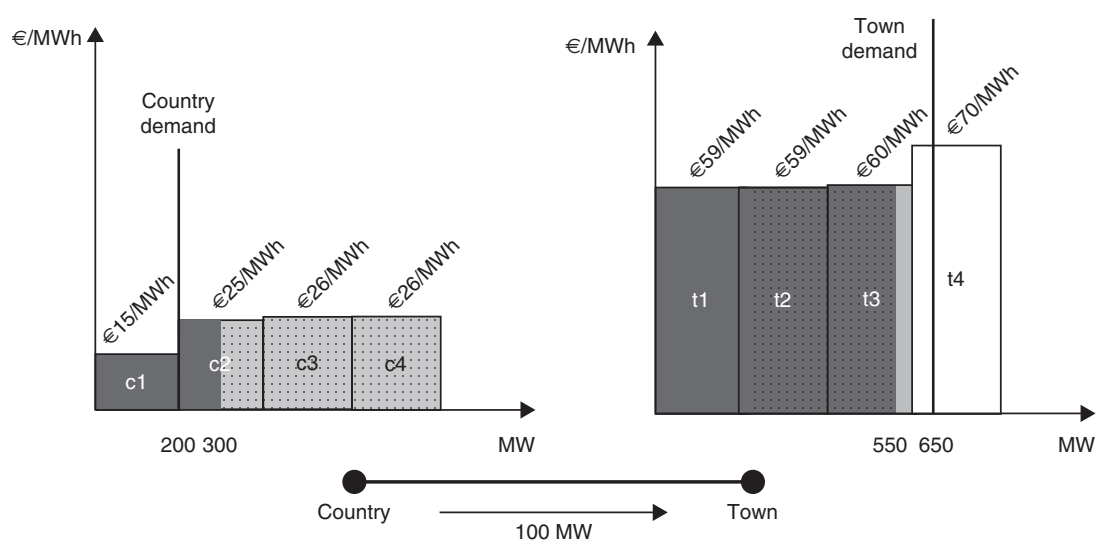

Figure 4.15 Outcome of the re-dispatch market

day-ahead price may change. In this example the marginal unit setting the day-ahead price becomes $t 1$ with its bid of $€ 59 / \mathrm{MWh}$. In the second place, the day-ahead dispatch results in a flow from the Country to the Town of $600 \mathrm{MW}$, requiring a re-dispatch of $500 \mathrm{MW}$, which is $200 \mathrm{MW}$ more than under the cost-based day-ahead bidding. This also increases the overall cost of re-dispatch as illustrated in Figure 4.15.

Three additional aspects of the incentives discussed above are worth mentioning: market power, auction design and congestion certainty:

- These bidding incentives arise in the case of perfect competition in each area of the market, and are not related to the exercise of market power. When exercising market power, generators bid differently from their variable production costs in order to modify the market price. When doing so they accept losing sales of a part of their capacity in order to receive increased profits from the remaining sales due to the higher prices. This market-power logic is not the one that drives the bidding incentives discussed above.

- The market outcome that we have discussed is to a large extent independent of the design of the day-ahead and re-dispatch markets. In the example we assumed that the re-dispatch market is run as a pay-as-bid auction. As discussed in Chapter 2, this means that the competitive generator in the import constrained area offers just below the highest price expected to ensure that an offer is accepted; similarly a competitive generator in the export constrained area forecasts the lowest price that would ensure that a bid is accepted and bid just above that price. In case the re-dispatch market were 
run as a non-discriminatory auction the bidding strategy of the generators would be different because accepted bids and offers respectively pay and receive the market clearing price, irrespective of the bid and offered prices. However, changing the auction design does not remove the fundamental incentive to arbitrage between the day-ahead and the re-dispatch prices.

- In this example we have assumed that generators have perfect information about the market, which allows them to accurately predict the competitive re-dispatch price in each area. In reality there exists a certain degree of uncertainty about the market parameters, which makes such prediction difficult. As a result, the competitive bidding strategies described above may present a certain degree of risk for generators. For example, if there is a high probability that the expected congestion will not occur, such bidding could on average result in losses rather than being profitable. The more uncertainty there is, the closer the day-ahead bids of competitive generators are to their variable cost.

\subsection{THE INTERFACE OR ZONAL APPROACH}

In this section we discuss the approaches to congestion management that use a simplified representation of the network, where network nodes are grouped in large zones and the constraints on individual transmission elements are grouped into interface constraints between zones.

These approaches mix the two approaches previously discussed. On the one hand, similarly to the nodal approach, transactions in the electricity market are constrained by the flow limits over the simplified representation of the network. On the other, if transmission congestion occurs within the zones defined by the simplified network representation, it is resolved using the re-dispatch approaches. The zonal approach has traditionally been implemented in Europe to allocate cross-border transmission capacity, in which case each country is considered a market zone.

Just as in the case of the nodal model, the rights to use transmission capacity over the simplified zone transmission model in the spot market can be defined explicitly and implicitly. Explicit rights between the exporting and importing zones allow spot transactions to be scheduled with power injections anywhere in the exporting zone matching withdrawals anywhere in the importing zone. Implicit rights are allocated through centralised clearing of the spot-market bids, and allow different spot prices to be set in the market zones in case the transmission flows over the interface constraints reach their limits. 

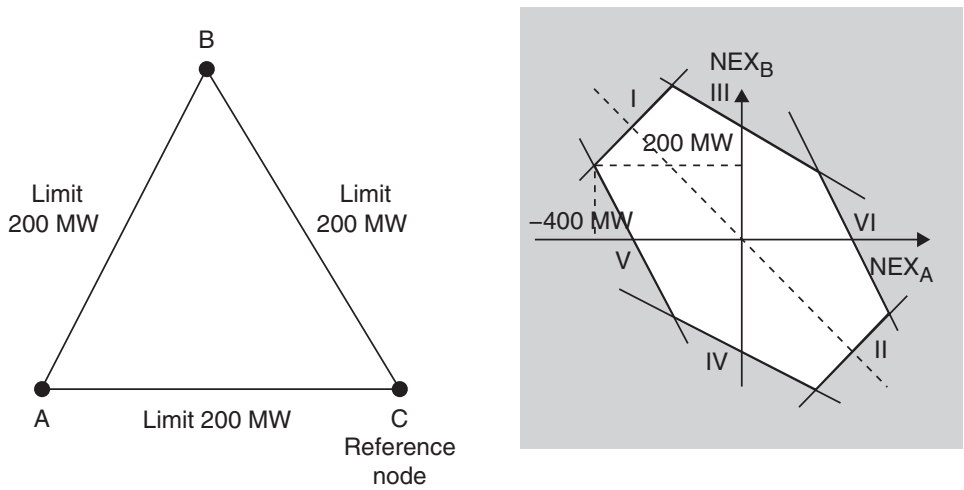

Figure 4.16 Transmission network and security domain in a 3-zone market

The approach used to simplify the real network into a zone representation is crucial in determining the amount of transmission capacity available between the market zones and for the resulting market outcomes. We discuss in the following sections two such approaches or capacity models: the net transfer capacity approach that has traditionally been used in Europe; and the flow-based approach that is currently being implemented in several regions of Europe.

\subsubsection{Capacity Model in a Three-market Security Domain}

In this and subsequent sections we discuss the zonal representation using a simple example of three countries: A, B and C, each country being a market zone. We first assume that the actual transmission network connecting the countries is rather simple (later in Section 4.5.3 we move away from this assumption) and similar to the triangular networks considered previously in this chapter. Each country is represented by a single node interconnected by transmission lines with identical impedance and an identical flow limit of $200 \mathrm{MW}$, as presented in the left panel of Figure 4.16.

First, we shall describe the characteristics of our network's security domain. The security domain is a combination of all possible sets of injections and withdrawals that do not violate any security constraints. In our three-market zone setting, each set of net withdrawals (conventionally called 'net exports' when referred to the zonal markets) from each market zone can be represented as a point in a two-dimensional space. The net exports in the third market zone chosen as the reference 
Table 4.4 PTDFs of a triangular network

\begin{tabular}{lcc}
\hline Line & $\mathrm{NEX}_{\mathrm{A}}$ & $\mathrm{NEX}_{\mathrm{B}}$ \\
\hline $\mathrm{AB}$ & $-1 / 3$ & $1 / 3$ \\
$\mathrm{BC}$ & $-1 / 3$ & $-2 / 3$ \\
$\mathrm{AC}$ & $-2 / 3$ & $-1 / 3$ \\
\hline
\end{tabular}

market will be uniquely determined by the balance condition, that is, the condition that the sum of the net exports in the three market zones is zero.

The right panel of Figure 4.16 shows the security domain in terms of the net exports from markets $\mathrm{A}$ and $\mathrm{B}\left(\mathrm{NEX}_{\mathrm{A}}\right.$ and $\left.\mathrm{NEX}_{\mathrm{B}}\right)$.

For this network, the PTDFs relative to the reference market $\mathrm{C}$ are shown in Table 4.4. As we discussed in the previous sections, the values of these PTDFs follow on from that fact that in this triangular network, for each MWh of transaction between two markets, the power flows split over parallel paths in the proportion of $2: 1$ according to the length of the parallel paths.

Each side of the security domain shown on Figure 4.16 represents the flow constraint on each transmission line. The constraint limiting the flow on line $\mathrm{AB}$ within $200 \mathrm{MW}$ in either direction identifies the set of net exports so that:

$$
-200 \leq \frac{1}{3} N E X_{A}-\frac{1}{3} N E X_{B} \leq 200 .
$$

This condition is satisfied by all combinations of $\mathrm{NEX}_{\mathrm{A}}$ and $\mathrm{NEX}_{\mathrm{B}}$ that lie in the region between lines I and II in the figure.

The flow constraint on line BC of $200 \mathrm{MW}$ in both directions identifies the set of net exports so that:

$$
-200 \leq \frac{1}{3} N E X_{A}+\frac{2}{3} N E X_{B} \leq 200 .
$$

This condition is satisfied by all points in the region between lines III and IV in the figure.

Finally, the constraint of $200 \mathrm{MW}$ on line AC in both directions identifies the set of net exports such that:

$$
-200 \leq \frac{2}{3} N E X_{A}+\frac{1}{3} N E X_{B} \leq 200 .
$$



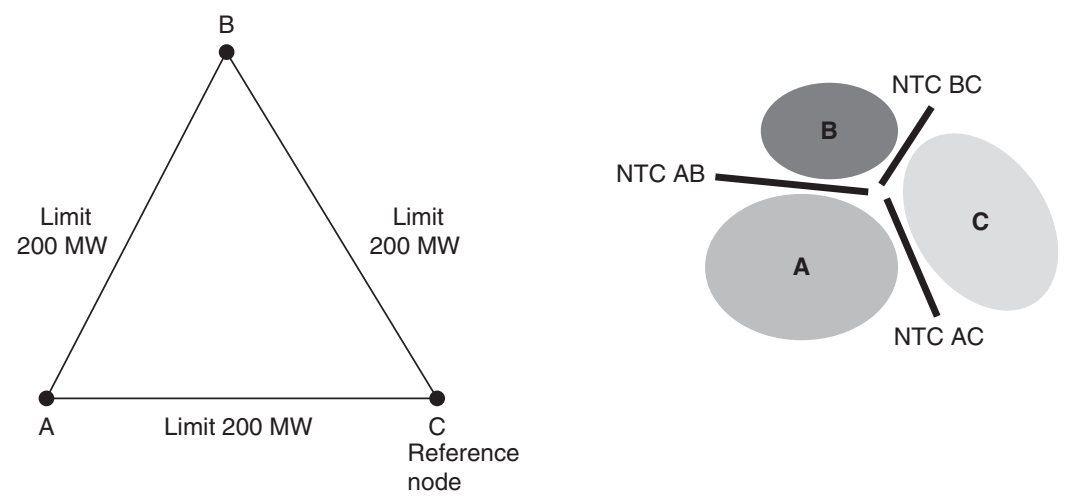

Figure 4.17 NTC transaction rights for a triangular network

This condition is satisfied by all net exports in the region between lines $\mathrm{V}$ and VI in the figure. The white surface in the figure above is the security domain of our network. Any set of net exports that lies within this area is feasible, since it meets all of the security constraints.

\subsubsection{The Net-transfer-capacity Capacity Model}

The traditional implementation of the interface methodology in Europe is commonly referred to as the 'net transfer capacity' (NTC) model. The main feature of the NTC model is that it sets limits on bilateral transactions between each pair of neighbouring countries regardless of the exchanges between the other countries. The NTC capacity model defines a matrix that determines the limits to bilateral trades between each pair of neighbouring markets (Figure 4.17).

At the initial stage of electricity market development in Europe, the system operators would issue and auction off (or otherwise allocate) explicit transmission rights corresponding to the NTCs on each border. These transmission rights would entitle the holders to schedule matching injections and withdrawals between the corresponding neighbouring countries.

However, this approach has often led to situations where available NTC capacity was not fully utilised in spot-market trades, or even utilised to schedule flows inconsistent with the spot-price difference, that is, implementing exports from the more expensive to the cheaper market. The efficiency of the cross-border transmission capacity utilisation was dramatically improved by the introduction of implicit auctions on some borders, where the allocation of cross-border NTC capacity is integrated with clearing of the spot markets. 
Table 4.5 NTC matrix

\begin{tabular}{lccc}
\hline From/to & A & B & C \\
\hline A & & 50 & 200 \\
B & 100 & & 150 \\
C & 250 & 200 & \\
\hline
\end{tabular}

The NTC capacity model is one of the ways to set limits on feasible commercial transactions, that is, to define a transaction space within the security domain. Using our simple example we analysed the transaction space provided by the NTC capacity model compared with the security domain. We highlight three important features of the NTC model:

- the same physical network can support multiple alternative NTC transaction spaces;

- a given feasible NTC transaction space may not be able to cover the entire security domain. Achieving some feasible transactions within the NTC model might mean not being able to achieve others; and

- in some cases there may be no feasible NTC transaction space that would allow the market to reach certain parts of the security domain.

A set of NTC limits on bilateral transactions between pairs of neighbouring countries (regardless of the exchange between other countries) implies that net exports from each country are limited by a simple sum of NTC limits between this and all the neighbouring countries. For example, consider the matrix of NTC limits in Table 4.5. This matrix implies that the maximum $\mathrm{NEX}_{\mathrm{A}}$ is given by the sum of $\mathrm{NTC}_{\mathrm{AB}}$ and $\mathrm{NTC}_{\mathrm{AC}}$ and is $250 \mathrm{MW}$, while the minimum $\mathrm{NEX}_{\mathrm{A}}$ is given by the negative of the sum of $\mathrm{NTC}_{\mathrm{BA}}$ and $\mathrm{NTC}_{\mathrm{CA}}$ and is $-350 \mathrm{MW}$.

Thus, the transaction space of the NTC capacity model can be presented in the form of individual limits to the net exports from each market. This space can be illustrated on a two-dimensional graph. Because the NTC capacity model sets individual limits, the zone net exports and the boundaries of the transaction space can only be represented by vertical lines, horizontal lines, or diagonals with an angle of $-45^{\circ}$. For example, the maximum and minimum limits on the $\mathrm{NEX}_{\mathrm{A}}$ are given by the vertical lines, the maximum and minimum limits on the $\mathrm{NEX}_{\mathrm{B}}$ are given by the horizontal lines, and the maximum and minimum limits on the $\mathrm{NEX}_{\mathrm{C}}$ are given by the diagonal lines. Along each of these diagonal lines the sum of $\mathrm{NEX}_{\mathrm{A}}$ and $\mathrm{NEX}_{\mathrm{B}}$ is constant and we know that 


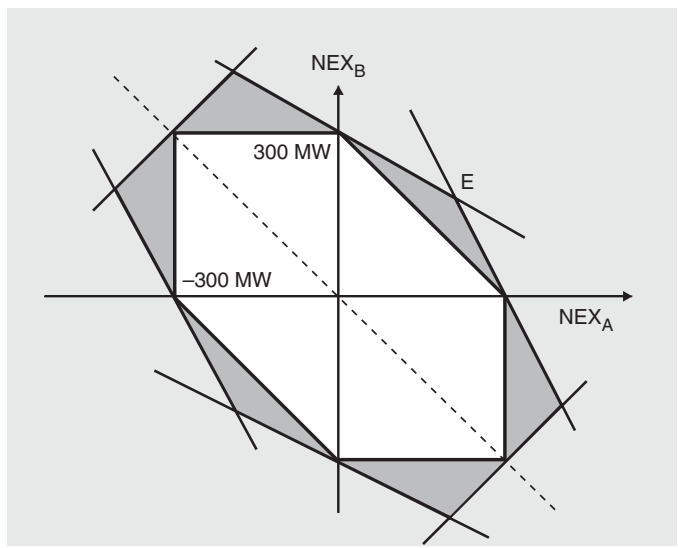

Figure 4.18 The largest NTC transaction space and the security domain

$\mathrm{NEX}_{\mathrm{C}}$ is equal to the sum of $\mathrm{NEX}_{\mathrm{A}}$ and $\mathrm{NEX}_{\mathrm{B}}$ taken with a negative sign. With these vertical, horizontal and diagonal lines, the shape of the NTC transaction space on a two-dimensional graph looks like either a square or a diamond. To make sure that any transactions on the market produce feasible transmission flows, the transaction space must lie entirely within the security domain.

Figure 4.18 illustrates the largest transaction space that can be supported by the security domain in our example. It is easy to verify that a vector of NTC levels making up this transaction domain is $150 \mathrm{MW}$ across each border in both directions. The figure shows that not all the feasible transactions can be achieved within this set of NTCs. For example, the transactions represented by point $\mathrm{E}$ in the figure correspond to $200 \mathrm{MW}$ imports into market $\mathrm{C}$ from market $\mathrm{A}$ and $200 \mathrm{MW}$ from market $\mathrm{B}$. This set of transactions is compatible with the network's capacity, as it results in a flow of $200 \mathrm{MW}$ on lines 13 and 23 and $0 \mathrm{MW}$ on line 12 . Nevertheless this set of transactions is not allowed by the selected NTC levels of $150 \mathrm{MW}$ on each border.

Note that one could choose a set of NTC values so that point E is included in the transaction space, for example: $200 \mathrm{MW}$ between zones $\mathrm{A}$ and $\mathrm{C}, 200 \mathrm{MW}$ between zones $\mathrm{B}$ and $\mathrm{C}$ and 0 between $\mathrm{A}$ and $\mathrm{B}$. The resulting transaction space is shown with a straight line in Figure 4.19. As shown in the figure, the inclusion of point $\mathrm{E}$ in the transaction space means excluding a large number of other feasible points belonging to the security domain. That means that when selecting the set of net transfer capacities allocated to the market, system operators must conjecture upfront which is the highest-value set of transactions. In our example, the system oper- 


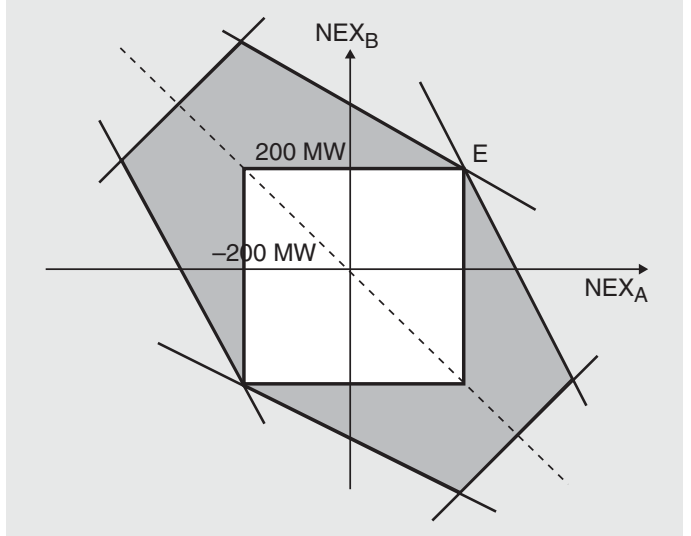

Figure 4.19 The NTC transaction space including point E

ators would select the NTC including point $\mathrm{E}$ in the transaction space if they knew that generation costs in market $\mathrm{C}$ are much higher than in both market $\mathrm{A}$ and $\mathrm{B}$, and that generation costs in market $\mathrm{A}$ and $\mathrm{B}$ are similar.

In the previous example we showed that a feasible transaction space defined by a given set of NTC limits might cover only a part of the security domain. In this case one can only select a transaction space that includes a specific point of the security domain at the expense of other feasible points, which would be put out of the market's reach.

In addition, in some cases, there is no choice in the NTC limits allowing market transactions in certain portions of the security domain. This happens because certain points of the security domain are only feasible when a particular transaction creating a counter-flow is scheduled. Consider the network shown in Figure 4.20. The transaction space shown in this figure as the area delimited by the solid dashed lines corresponds to the following set of NTC limits: $\mathrm{NTC}_{\mathrm{BC}}$ and $\mathrm{NTC}_{\mathrm{AC}}$ are $600 \mathrm{MW}$, while $\mathrm{NTC}_{\mathrm{AB}}, \mathrm{NTC}_{\mathrm{BA}}, \mathrm{NTC}_{\mathrm{CB}}$, and $\mathrm{NTC}_{\mathrm{CA}}$ are all zero. One can interpret this selection of net transfer capacity as aiming to maximise imports into (or exports from) Country C. According to the figure, a large part of the security domain does not belong to the transaction space. In particular, a number of transactions that the network can support and that would result in greater imports into $\mathrm{C}$ are not allowed. Yet it is impossible to expand the transaction space any further. Point E, for example, in which market A exports $900 \mathrm{MW}$ towards market C, is feasible only if a counterflow transaction from B to C of $300 \mathrm{MW}$ is scheduled. However, in the NTC model there is no means of identifying such a condition since each transaction is considered independently from the others. 

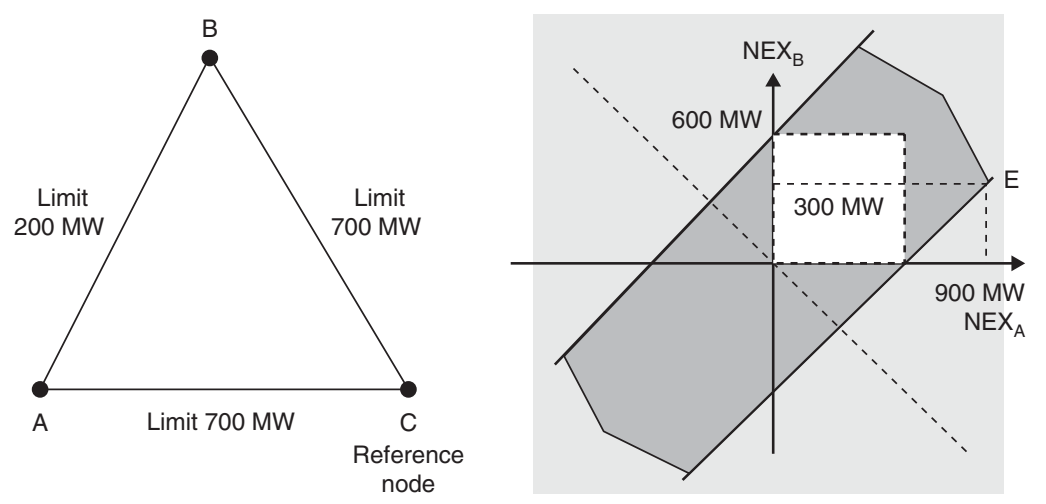

Figure 4.20 Transaction space of the explicit flow-based auction

Therefore, because of its simplicity the NTC model is unable to allow market participants to explore the entire security domain.

\subsubsection{The Flow-based Capacity Model}

The development of power exchanges in many European countries and increasing coordination among system operators have paved the way for the introduction of a new capacity model that is potentially more efficient than the traditional NTC approach. This new methodology is commonly referred to as 'flow based'.

In the flow-based approach, each constraint sets a limit to the flow over a critical infrastructure or critical branch, a physical transmission network element affected by the cross-border transactions. This constraint then sets a ceiling to the weighted sum of the net exports from all market zones, where the weights are the PTDFs of these market zones with respect to each critical branch. The constraints have a similar structure to those discussed in Section 4.3:

$$
\sum_{i \neq r} P T D F_{i j}^{r} \cdot N E X_{i} \leq F L_{j}
$$

Thus the set of flow-based constraints and the transaction space defined by the flow-based capacity model coincide with the security domain. In the example presented in Section 4.5.1, the flow-based transaction space would match the security domain shown in Figure 4.16.

This represents a major improvement on the NTC model where, as we saw above, the transaction space may cover only parts of the security domain. For this reason, use of the flow-based capacity model is expected 
to allow larger volumes of cross-border trade and a more efficient use of the existing physical network in general.

Currently two versions of flow-based methodology are being developed in Europe. One is flow-based market coupling between France, Belgium, the Netherlands, Germany and Luxembourg (the Central West Europe region, or $\mathrm{CWE}$ ). In the $\mathrm{CWE}$, the day-ahead electricity markets of the participating countries are simultaneously cleared in such a way that the trade surplus is maximised subject to flow-based constraints. ${ }^{8}$ The rights to use the transmission system are thus implicitly allocated, as we discussed in Section 4.3.2.

The second version of flow-based methodology is being developed for the allocation of explicit short-term transmission rights between Austria, the Czech Republic, Germany, Hungary, Poland, the Slovak Republic and Slovenia (the Central-East Europe region, or CEE). In this model, the markets for energy and for transmission rights are not cleared simultaneously. First market participants submit bids for transmission rights between any pair of participating national markets. The auction for these rights is cleared in such a way that the value of the awarded transmission rights is maximised subject to the set of flow-based constraints, ${ }^{9}$ similarly to the FTR allocation process in the nodal markets discussed in Section 4.3.3. The transmission rights allocated in this process give their holders the rights to schedule matching transactions between market zones in the day-ahead market.

The transaction space provided by the explicit version of the flowbased capacity model should be expected to be smaller than the one that the implicit model could achieve. This is because the transmission rights allocated in the explicit model have the character of an option. As we discussed in Section 4.3.3, such option rights may not be capable of fully utilising available transmission capacity or fully exploiting the counterflows that can increase transmission capacity.

\subsubsection{Accounting for Nodal Details in the Flow-based Capacity Model}

The interface approach addresses network constraints by limiting net power transfers between large areas of the network, or zones. In the examples discussed thus far we have assumed that each market zone could realistically be approximated by a single network node. In other words we assumed that, power injections at any part of the market zone have the same impact on flows over all the critical branches.

In this section we address issues related to the actual size of the zones in the context of the interface approach. Specifically we consider situations where net injections at different nodes of the same zone could cause 


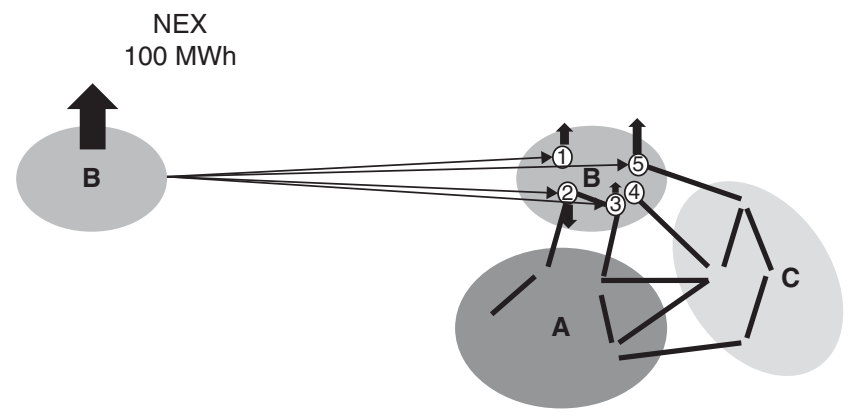

Figure 4.21 The link between the net exports and nodal injections through GSKs

different flows across critical infrastructures. This could happen in a wide range of situations, especially when a single market zone comprises a large country. This may happen, for example, when a critical infrastructure is located entirely within the zone. In this section, we refer to the most efficient version of the interface approach, flow-based market coupling. However, similar issues arise in all other implementations of the interface approach.

When different nodes in a zone have a different impact on a critical branch, the same value of the total net exports from the zone can have a different impact on the critical branch, depending on how the net exports are distributed among the nodes of that zone.

In Section 4.3, above, we noted that the impact of each node on the flow over a constraint is given by a PTDF. In the flow-based approach, such node-level PTDFs are synthesised into zone-level PTDFs taking weighted averages of the node-level PTDFs over all nodes belonging to a given zone. The nodal weight factors used in this process are called 'generation shift keys' (GSKs). ${ }^{10}$

The GSK of each node is the share of the zone's net injections that is expected to take place in that node in real time. Figure 4.21 illustrates the link between the net exports from the zone and the corresponding nodal injections through GSKs. The zone-level PTDF for a critical branch is then calculated by summing the PTDFs for that branch of all nodes belonging to the zone, weighted by the GSKs:

$$
\begin{gathered}
P T D F_{z j}=\sum_{i \in z} P T D F_{i j} \cdot G S K_{i}^{z}, \\
\sum_{i \in z} G S K_{i}^{z}=1 .
\end{gathered}
$$


If the actual distribution of injections across the nodes grouped in the zone is different from the expected distribution reflected in the GSKs, the actual flows over the critical branches will be different from the flows implied by zone-level PTDFs. The same level of net exports from the market zone can induce an unexpected flow over the critical branches if the actual distribution of nodal net injections diverges from the one reflected in the GSKs. Specifically, the actual flows over a critical infrastructure may turn out to exceed the security limit even if the net injections meet the constraints defined at the zone level. This possibility is dealt with by reducing the capacity of the critical infrastructure made available to the market by the flow reliability margin (FRM) ${ }^{11}$ The system operators assess the variation in the flow over each critical branch resulting from errors in the GSK prediction and use the extreme values of this variation to set the FRM for the branch.

Application of the FRM reduces the transaction space compared with the security domain achievable under the nodal approach. The FRM and reduction of the trading space are all the more significant for large national markets in which transmission nodes in different parts of the country have very different impacts on the flows over the cross-border critical branches. ${ }^{12}$

The choice of GSKs may also have an important impact on the market outcome in the flow-based approach. In Section 4.3 it was shown that the locational market-clearing price of electricity may reflect the marginal cost of congestion that incremental demand in this location creates or resolves in other parts of the network. The GSKs may have an impact on the valuation of that impact on congestion, often providing a biased valuation.

We illustrate this effect in the usual three-zone market. The setting of our example is illustrated in Figure 4.22. We assume for simplicity that in

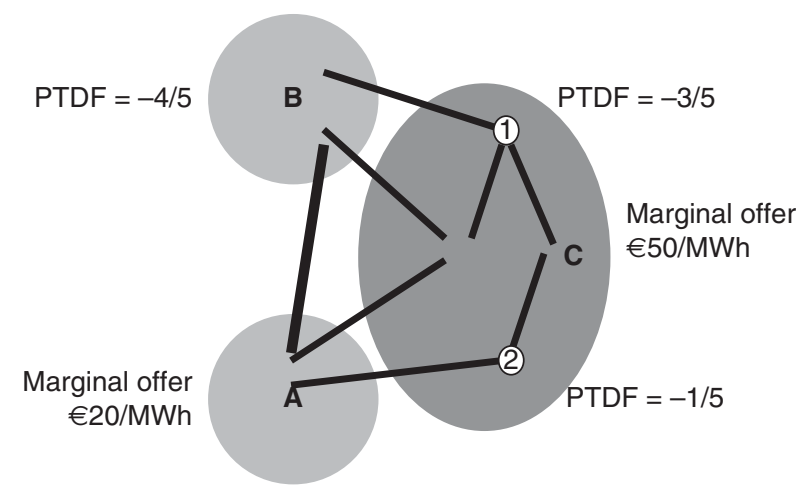

Figure 4.22 Impact of GSKs on market prices 
zones $\mathrm{A}$ and $\mathrm{C}$ unlimited generation capacity is available at variable costs of $€ 20 / \mathrm{MWh}$ and $€ 50 / \mathrm{MWh}$, respectively, and that in equilibrium the link between market zones A and B is constrained, while all remaining critical branches have ample capacity. In this example we also assume that zone $\mathrm{C}$ consists of two nodes that have a very different impact on the binding constraint. The PTDF of node 1 calculated relative to the reference market $\mathrm{A}$ is $-3 / 5$ (close to the PTDF of market $\mathrm{B},-4 / 5$ ), while node 2 has a PTDF of $-1 / 5$ (close to the PTDF of market A, 0 ). In order to isolate the effect on the market outcome of the methodology to set the GSKs, we assume that the market outcome can be perfectly predicted by the system operator. This rules out any distortions related to prediction errors.

Under our assumptions, the market equilibrium prices in zones A and $\mathrm{C}$ are equal to the marginal generation costs, $€ 20 / \mathrm{MWh}$ and $€ 50 / \mathrm{MWh}$, respectively. Given the zone-level PTDFs, the market-clearing price in zone B can then be determined through the relationship, discussed in Section 4.3:

$$
P_{z}=P_{r}-\sum_{j} P T D F_{z j}^{r} \cdot S P_{j}
$$

For each selection of GSKs one set of zone-level PTDFs and therefore one price level in B are determined. We consider three cases of GSKs, one that puts 100 per cent of the weight on node 1 , one that puts 100 per cent of the weight on Node 2, and one that weights the two nodes equally. Table 4.6 shows calculation of the prices in market B for these GSK cases.

The zonal PTDFs corresponding to the three GSK scenarios are 3/5, $1 / 5$ and $2 / 5$, respectively. The constraint shadow price can be calculated for each value of zonal PTDF as the difference between the prices set by generators in zones $\mathrm{C}$ and $\mathrm{A}, € 30 / \mathrm{MWh}$, divided by the PTDF of zone $\mathrm{C}$. Finally, the price in market $\mathrm{B}$ in each scenario is calculated using the above formula, with the prices in reference zone $\mathrm{A}$, the constraint shadow price, and the PTDF of zone B.

The table shows that the price in B is highly dependent on the choice of GSKs. Prices would reflect the correct marginal cost of congestion created

Table 4.6 GSK impact on market prices

\begin{tabular}{|c|c|c|c|c|c|}
\hline \multirow[t]{2}{*}{ GSK } & \multicolumn{2}{|c|}{ GSK weights } & \multirow{2}{*}{$\begin{array}{l}\text { PTDF of } \\
\text { zone C }\end{array}$} & \multirow{2}{*}{$\begin{array}{l}\text { Constraint } \\
\text { shadow price }\end{array}$} & \multirow{2}{*}{$\begin{array}{c}\text { Price } \\
\text { market B }\end{array}$} \\
\hline & Node 1 & Node 2 & & & \\
\hline 1 & 1.0 & 0 & $-3 / 5$ & 50 & 60 \\
\hline 2 & 0 & 1.0 & $-1 / 5$ & 150 & 140 \\
\hline 3 & 0.5 & 0.5 & $-2 / 5$ & 75 & 80 \\
\hline
\end{tabular}


by the demand in each zone if, in each zone, the node where the marginal plant is located receives GSK weight 1. Such marginal GSK weighting is very difficult to implement, since it is much more difficult to predict the marginal plant than the average contribution of each node to the zone's production. However, in most cases the average GSK proportional to actual production in each node will produce zonal prices incorrectly reflecting the marginal cost of congestion. This may result in inefficient distribution of production across the zones. In our example, the market equilibrium production level in B could be materially higher if the clearing price was $€ 140 / \mathrm{MWh}$ rather than if the price was $€ 60 / \mathrm{MWh}$.

Furthermore, even though GSKs related to the marginal units could provide the efficient price outcome, such GSKs may result in incorrect assessment of the flows produced by the net exports from each market zone over the critical branches. These flows depend on the net injections in all nodes, and not only on the injections at the marginal node. Only average GSKs that are proportional to the actual nodal production and the actual nodal share of the total zonal export correctly predict the flows on the critical branches that will result in market equilibrium. Using marginal rather than average GSKs may then require larger FRM, to the detriment of the transaction space.

Perfect GSKs, which would both lead to efficient prices and allow correct assessment of the power flows over critical branches, do not exist.

\subsection{THE LOCATIONAL PRICE AND RE-DISPATCH DEBATE}

In this section we present some elements of the longstanding debate about the pros and cons of the approaches to congestion management discussed in Sections 4.3 and 4.4, above: the nodal and the re-dispatch approaches. We also refer to current European developments in zonal approaches that to some extent mix the elements of the nodal approach, in the flow-based market coupling of market zones, and the re-dispatch approach to congestion within the zones. The debate is ongoing in the context of European electricity market integration, particularly with respect to of the optimal size of the market zones in flow-based market coupling.

We discuss the following aspects of the debate: the cost of congestion, market power, market liquidity, implementation and operation complexity, incentives for generator investments, incentives for network development, political pressure for a single national price for customers, and the wealth distribution properties of the two approaches. 


\subsubsection{Cost of Congestion}

Nodal congestion management has been argued to result in a higher cost of congestion eventually paid by market participants than the alternative single price system with re-dispatch. Several formulations of this critique have been brought forward. ${ }^{13}$ These critiques can be illustrated by a simple example, where a generator with a very high cost located in an importconstrained area has to be turned on to produce a small amount of energy in order to relieve congestion. In the nodal approach, this generator considerably increases the clearing price that is paid by all customers and received by all other generators in this location. It is argued that if the same congestion was dealt with using the re-dispatch approach, the impact on the market would be much smaller, since the cost of re-dispatch would amount only to the cost of the additional power from this generator.

This reasoning has several flaws. First, it neglects the congestion rent that is collected by the system operator in the nodal approach when there is congestion, which is eventually returned to market participants. Part of the increase in the locational price in the import-congested area will therefore be hedged by the congestion rent. The total cost of congestion to customers after the congestion rent is passed on to them may be similar to the cost in a re-dispatch approach.

Second, this example assumes that in the re-dispatch system competitive generators would offer their capacity at variable cost. However, this is not the case, as discussed in Section 4.4. Because of the day-ahead bidding at opportunity cost induced by re-dispatch congestion management, customers could eventually face a higher cost of congestion than in the nodal approach. On the other hand, the nodal market clearing provides an incentive to competitive generators to offer their power at marginal cost and does not induce any opportunity cost bidding.

A more substantial argument on the cost of congestion relates to markets where transmission congestion has to be addressed by the re-dispatch approach in time, through balancing market transactions. Performing such real-time re-dispatch may turn out to be unnecessarily expensive for the system operator, because in real time it may have a limited choice of fast and flexible generating resources available to provide re-dispatch. Congestion could be resolved more cheaply if it were addressed at an earlier stage, for example, in the day-ahead market, where there is enough time to start up and ramp up inflexible units that could deal with congestion most efficiently.

As the volumes of re-dispatch become important and the need to start up large inflexible units for re-dispatch purposes increases, the inefficiency of dealing with re-dispatch in real time becomes more and more apparent. 
In several US markets, such as California and Texas, one of the main reasons for transition to the nodal market design was the excessive cost of real-time system re-dispatch.

\subsubsection{Market Power}

The problem of market power in electricity markets can be exacerbated by the presence of transmission constraints. Transmission constraints create fragmented geographical markets with less competition than on a broader market level. For instance, when transmission constraints are binding in the direction of a large load centre, this centre becomes a separate market, or a load pocket. Generators outside the load pocket cannot exert competitive pressure on the generators inside the pocket, making market concentration in the load pocket higher than in the market as a whole. ${ }^{14}$

One of the common critiques of the nodal system is its perceived higher vulnerability to locational market power than a system based on redispatch. This perception is incorrect. The ability of generators to exercise locational market power depends on the topology of the transmission network, and not on the choice of congestion management mechanism. The congestion management system only determines how market power is exercised and the mechanism by which it impacts customers.

In the locational price system, market power is exercised through the submission of supply bids above variable costs with the intention of increasing locational prices. In re-dispatch systems, market power is exercised through the inflation of re-dispatch payments. This can be done by first submitting day-ahead bids that ensure that a generator participates in the re-dispatch market, and then submitting re-dispatch bids in excess of the re-dispatch equilibrium price. Since the re-dispatch costs are socialised and averaged over all system users, it is more difficult to observe the impact of the exercise of market power in the re-dispatch system.

The exercise of locational market power in re-dispatch systems involves day-ahead and re-dispatch bidding that is similar to the strategies followed by competitive generators exercising arbitrage opportunities in the presence of constraints, as described in Section 4.4, above. In both cases, generators do not bid according to their production costs. However, as opposed to competitive generators, bids by generators exercising locational market power exceed the re-dispatch competitive equilibrium price. Yet the similarity of the competitive and abusive bidding strategies makes it more difficult to distinguish the exercise of market power from competitive behaviour in the re-dispatch system than in the system with locational prices, where competitive generators bid at variable cost.

The re-dispatch approach cannot create more competition among 
generators by assuming no constraints in the day-ahead market. Instead, it tends to obscure the market power that is exercised in the subsequent redispatch market. In contrast, the locational price-based market does not create market power, but makes it more transparent. ${ }^{15}$

There is one important case where the re-dispatch and locational price systems may impact differently on the opportunity for generators to exercise market power. This is market power in the export-constrained area. In the re-dispatch system, a generator with such market power can set exportside re-dispatch prices at very low or negative values and achieve profits equivalent to the difference of this price from the day-ahead price. In the locational price system, such a generator would also be able to set a low or negative locational price, but there is little or no opportunity to convert this low energy price into profits.

\subsubsection{Market Liquidity}

It is sometimes argued that a system with locational prices may reduce the liquidity of wholesale power markets. The argument suggests that whereas in a re-dispatch system a market participant (for example, a retail supplier looking to buy power) could look for a counterparty among operators in the entire national market, in the locational price system it should either look for a counterparty within a smaller area or pay congestion price differentials if the counterparty is found in a wider area.

This argument tends to misinterpret the implications for market liquidity of both the locational price system and the re-dispatch system.

In the system of locational prices, counterparties for bilateral trades (for example, using contracts for differences) can still be found throughout the entire market. In the event of transmission congestion the transaction would incur a congestion cost equivalent to the difference between clearing prices in the sink and the source area of the trade. This congestion cost can be hedged by acquiring the FTRs between the production and the consumption nodes matching the trade. Thus, the system of locational prices does not limit the scope of bilateral trades and provides the means to hedge such trades.

The systems featuring a day-ahead market with a nationwide uniform price and subsequent re-dispatch may appear to provide more opportunities for bilateral trades throughout the entire territory. However, it is often not considered that in the event of congestion such a system can provide only a partial hedge, since no financial hedge against the volatility of the tariff component covering re-dispatch costs is generally available at market participants. Thus, in the event that re-dispatch costs are material, the re-dispatch system may impede bilateral trades 
because of its inability to provide a hedge for a significant wholesale cost component.

\subsubsection{Implementation and Operation Complexity}

A common critique of the nodal congestion management system as compared with the single-price re-dispatch approach is based on the argument that the process of calculating nodal prices in the presence of congestion is very complex and non-transparent, undermining market participants' confidence in these markets.

However, the calculation of nodal prices follows the logic of constrained optimisation algorithms, and the resulting prices meet a certain number of high-level properties, such as those presented in Section 4.2, above. Such high-level properties can be verified and audited.

Although the calculation of nodal prices is indeed more complex than the calculation of a single unconstrained clearing price, it is certainly not more complex or less transparent than the subsequent calculation of nodal re-dispatch instructions and payments that needs to be performed in the single-price system in the presence of congestion. Furthermore, as discussed in Section 4.4, above, bidding incentives induced by the re-dispatch congestion management may also affect preceding markets, such as the day-ahead market. Therefore, confining the nodal clearing system to the re-dispatch phase cannot be considered to limit the impact of its complexity to a narrow segment of the market.

\subsubsection{Incentives for Network Development}

It is often argued that because the system of locational prices allows the system operator to collect congestion rent, it provides incentives to the system operator to retain congestion rather than to reduce it through short-term measures or through building transmission upgrades.

However, in no existing system does the system operator appropriate the congestion rent as additional profit. The congestion rent is generally returned to market participants in one form or another. Thus the congestion rent collected by the system operator in the process of the congestion management mechanism does not have an impact on the system operator's incentives to retain congestion through short-term actions or to delay necessary transmission investments.

Regulatory incentives for the system operator to make timely investments in transmission infrastructure can be put in place regardless of the chosen method of congestion management. The regulatory incentives to invest in transmission can be based on the objective indicators of the social 
benefit of the investment, which does not depend on the chosen congestion mechanism.

\subsubsection{Incentives for Generation Investment}

Systems featuring re-dispatch congestion management and systems featuring locational prices provide different incentives for investment in generation capacity.

The re-dispatch system aims to maintain uniform price signals over the entire area. This system may give little additional incentive for generator incentives in import-constrained areas where additional capacity is most needed. On the contrary, it may induce entry in areas where there is already excess capacity and from which export is limited. New generation capacity in these areas will seldom be activated and will more often be collecting re-dispatch payments for being constrained off. This may also provide an adverse incentive for investment in inefficient plant.

Generation investment induced in re-dispatch systems further increases the need for and cost of re-dispatch.

On the other hand, systems where congestion is resolved by setting different market prices in different locations indicate the marginal value of energy in a given location given the transmission constraints. These prices provide more precise signals of the need for generation investment in different geographical areas. Locational spot-market prices can be further hedged in forward markets, and locational differences can be hedged with long-term transmission contracts, FTRs, providing market participants with the necessary instruments for mitigating investment risks.

\subsubsection{Political Pressure for a Single National Price for Customers}

Congestion management via locational prices implies that different customers may pay different electricity prices just because of their location. That feature has been the source of political opposition to locational prices, since price uniformity for essential goods such as electricity has traditionally been a social policy objective.

However, congestion management via locational prices may be implemented while preserving the traditional cross-subsidies among customers located in different geographical areas, through a proper market design. This happens in several US markets in which prices charged on withdrawals are uniform within each zone, and equivalent to the average of the nodal prices in the zone. In Europe the same logic applies in Italy, where withdrawals are charged at a nationwide uniform price, while generators are paid geographically differentiated prices. 
As long as the electricity demand is price inelastic, ${ }^{16}$ congestion management systems based on asymmetric prices for generation and load do not give rise to major inefficiencies, since charging loads the uniform price or the nodal price does not alter the purchased volumes. Inefficiency caused by the lack of locational signals on the demand side is more likely to appear in a longer-term horizon. Specifically, uniform demand-side prices could distort the siting decisions of large industrial customers.

\subsubsection{Surplus Distribution Properties}

Markets with locational energy prices and markets based on re-dispatch have very different surplus distribution properties. Whereas the locational prices reflect the marginal value of energy at each location, re-dispatch systems intend to maintain generators' revenues at the level they would be if there were no congestion. The re-dispatch system therefore creates cross-subsidisation among market areas, maintaining the price above the marginal value of energy in the area in export-constrained areas and the price below the marginal value of energy in import-constrained areas.

Furthermore, if congestion is predictable, arbitrage between the energy and the re-dispatch market may lead to a uniform market-clearing price higher than that without congestion, as we discussed in Section 4.4.2. In this case the re-dispatch system results in greater total supply costs for the consumers, compared with the nodal approach.

Although the transition from a re-dispatch market to a market with locational price differentiation can be expected to improve market efficiency overall, there may be both winners and losers as a result of this transition. Parties that may be disadvantaged by the locational system are generators located in export-constrained areas that benefit from the re-dispatch system, which allows them to maintain a price above the marginal value of energy in their location.

For these parties, transition to the locational price system can be made more acceptable by allocating them the FTRs between their exportconstrained area and the remaining areas.

\subsection{NETWORK DEVELOPMENT}

Transmission and generation capacity are linked by complex relations of complementarity and substitutability. A network upgrade may be necessary in order to transfer a new generator's production from the injection node to the load centres. Alternatively, a transmission upgrade might make it possible to meet a demand increase at a certain location with 
production from existing generators at other locations, thus avoiding the construction of additional capacity near the load centre. Thus, total supply cost minimisation requires transmission and generation investment decisions to be coordinated.

In most electricity markets, the system operator is the monopoly supplier of transmission services. The system operator is responsible for planning the network's upgrades in order to increase the transmission capacity. Where generation is liberalised, private investors decide when and where new generation plants will be built. In this setting the network development decisions are not made by those who bear their economic consequences, that is, the power generators and consumers.

Coordination between transmission and generation investment decisions is different depending on the congestion management system implemented in the market. Where congestion management is carried out via re-dispatch, the economic consequences of transmission investments are borne mostly by electricity consumers. They pay transmission charges, ${ }^{17}$ ensuring full recovery of the network costs irrespective of the actual use of the transmission capacity. This means that if the system operator's decisions lead to overinvestment, consumers pay for unnecessary transmission capacity. In the event of underinvestment, inefficiently high re-dispatch payments are made to the generators and passed on to the consumers via the transmission tariffs. As we discussed in Section 4.4, in a re-dispatch system generators do not bear any cost in the event of congestion, since the re-dispatch payments are such that constrained-off generators obtain at least the same profits as if there were no congestion. ${ }^{18}$

Since in this setting a generator's profit does not depend on its location, ${ }^{19}$ the sites where new capacity is built might be inefficiently selected. In practice there are ways for the system operator to influence generators' localisation decisions, for example by denying or delaying interconnection at the nodes where additional injections would create congestion. This may reduce the scope for the inefficient localisation of generation plants. However, the transmission and generation investment decisions are coordinated by an administrative process, rather than via the pricing system.

In the event that congestion management is carried out via locational prices, both generators and consumers are affected by the system operator's network investment decisions, via the impact of network upgrades on electricity prices at the different locations, as well as via the transmission tariffs. In the event of underinvestment, electricity prices in some areas will be higher than optimal and some efficient generation capacity in some areas will not be activated (without being compensated). In the event of overinvestment consumers pay larger than necessary transmission tariffs.

In this setting the generation localisation decisions internalise the 
expected network upgrades via their impact on electricity prices at the different locations. For example, if the system operator's network development plan does not include upgrades relieving a certain constraint, the expectation of high prices at the import side of the bottleneck will attract new generation investment in that area. However, even in this context, coordination between transmission and generation investments is not fully market based. The system operator's views on the (efficient) future geographical distribution of the generation fleet - reflected in its network development decisions - may be different from the market's views. When this happens, there is no mechanism allowing one party to take control of the network investment decisions and assume the corresponding risk.

In an alternative approach to network development, market investors take the risk of expanding transmission capacity. They pay for network upgrades and become owners of the transmission rights that result from the upgrades. This model is often referred to as a 'merchant model', and we discuss it in the framework of a locational price congestion management system. ${ }^{20}$ A simple example can be used to illustrate how coordination between generation and transmission decisions takes place in a merchant context. Figure 4.23 illustrates our example.

The prices shown in the figure are for a base-load future contract with delivery at each node. The price at node 1 is lower than at node 3 . As we have seen in Section 4.3.2, upgrading the capacity of the line 1-3 by $100 \mathrm{MW}$ allows the system operator to issue an additional $150 \mathrm{MW}$ of financial transmission rights between node 1 and node $3 .{ }^{21}$ In the merchant approach any investor would be allowed to build the network upgrade. ${ }^{22}$

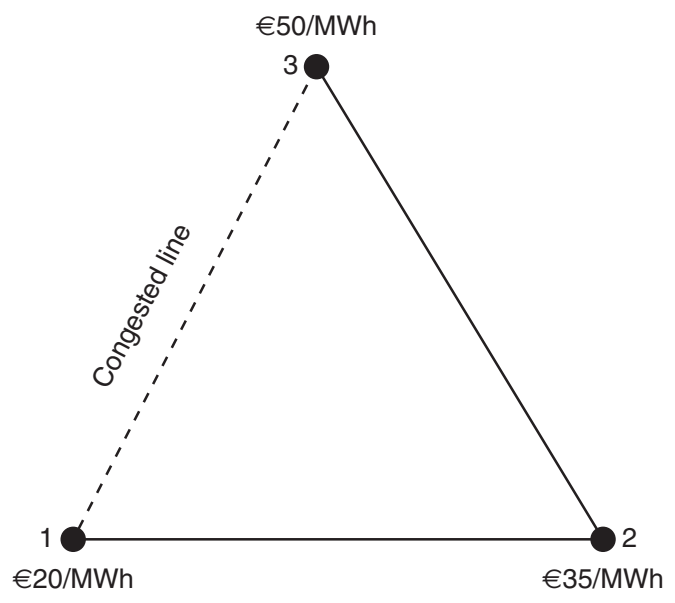

Figure 4.23 Triangular network 
In exchange for each MW of the upgrade, the investor is granted 1.5 MW incremental financial transmission rights from node 1 to node 3 at no charge over the economic life of the upgrade. ${ }^{23}$ The entire network capacity continues to be allocated on a short-term basis through the spot energy market, as shown in Section 4.3.

The investors' revenues come from exercise of the financial transmission rights. For each MW the transmission rights holder collects the price difference between nodes 3 and 1 . The network capacity between nodes 1 and 3 is increased to the expected electricity prices, such that the value of a $1 \mathrm{MW}$ transmission right between nodes 1 and 3 equals the incremental cost of expanding the capacity of line $1-3$ by $2 / 3 \mathrm{MW}$.

In this context, coordination between generation and transmission investments takes place according to the standard market mechanism. Rational investors will compare the profitability of building additional generation capacity at node 1 and of increasing transmission capacity between nodes 1 and 3. Either strategy can be hedged by taking appropriate forward electricity positions. The investment in generation capacity at node 3 can be hedged by selling power at node 3 . The investment in transmission capacity can be hedged by purchasing electricity at node 1 and selling the same quantity at node 3 .

The lumpy nature of network investments is a potential source of inefficiency of the merchant approach. In our setting, for example, assume that the only technically feasible upgrade of line 1-3 is such that congestion is completely eliminated. After the upgrade, the electricity prices at nodes 1 and 3 will therefore be identical. Assume also that implementing the upgrade is efficient, that is, that the net surplus generated by the additional electricity transaction enabled by the upgrade is greater than its cost. Despite its positive net value, no investor would want to invest in the network upgrade, because it would result in an excess supply of transmission capacity, which would bring the value of the transmission rights from node 1 to node 3 down to zero. This is the result of the combined effect of the upgrade's lumpiness and of the impossibility of limiting the physical power flows over the network. Intuitively, once the lumpy upgrade is in place the owner cannot limit its use in order to prevent convergence of the nodes 1 and 3 prices. ${ }^{24}$

Furthermore, the merchant approach might not be viable if hedging the investments in network upgrades were made impossible by the lack of liquidity of the forward electricity markets.

In practice, the merchant approach could be undermined also by the difficulty of assessing the value of transmission rights granted to those investing in network upgrades. Without a detailed knowledge of the network operations, potential network investors might find it difficult to assess the 
impact of further transmission and generation investments on the value of their transmission rights.

In Europe a planning approach to transmission network development is implemented in all countries. Merchant cross-border investments are only allowed, as an exception to the default regime, for DC interconnectors. Exemption is granted on a case-by-case basis and only if certain conditions are met. ${ }^{25}$ Coordination issues between merchant projects and network developments planned by system operators have not surfaced so far, presumably because of the limited scope of the merchant initiatives.

\section{NOTES}

1. To a limited extent the system operator can change the network topology, and thus the flows over network elements, by opening or closing circuits or by changing the setting of devices called 'phase shifters'. We abstract here from that possibility.

2. On how losses and non-linearity are accounted for in power flow modelling see, for example, Hogan, W.W., Pope, S.L. and Harvey, S.M. 1997. 'Transmission Capacity Reservations and Transmission Congestion Contracts', Center for Business and Government, Harvard University, 8 March.

3. For the time being we have assumed that there are no flow limits on the other lines.

4. Proponents of this approach suggest that those rights should be defined only for potentially congested network elements (Chao, H. and Peck, S., 1996. 'A Marker Mechanism For Electric Power Transition', Journal of Regulatory Economics, 10, 25-59).

5. We shall discuss in greater detail the determination of the feasible set of transmission rights in Section 4.3.2, in the context of the point-to-point definition of the transmission rights.

6. Kristiansen, T., 2004. 'Markets for Financial Transmission Rights', Energy Studies Review, 13(1), 25-74.

7. The same market-clearing algorithm is implemented in the real-time market.

8. CWE Orientation Study, 2008. A Progress Report for the MoU Signatories on the Design of the Flow Based Market Coupling in the Central West European Region, CWE MC Project, February.

9. Central Allocation Office and Consentec, 2009. Concept of the Technical Parameters Calculation for the Flow Based Capacity Allocation in the CEE Region.

10. Ibid.

11. Ibid.

12. The logic underlying the FRM is similar to that of the transmission reliability margins (TRMs) featuring in the traditional NTC implementation of the interface approach.

13. See, for example, Rosenberg, A.E., 2000. 'Congestion Pricing or Monopoly Pricing?', The Electricity Journal, 13(3), April, 33-41.

14. In fact, constraints do not even have to be binding for locational market power to become an issue. In the event of a price increase by a generator located inside a load pocket, the mere possibility of binding transmission constraints may itself trigger the exercise of locational market power (see, for instance, Borenstein, S., Bushnell, J. and Stoft, S., 2000. 'The Competitive Effects of Transmission Capacity in a Deregulated Electricity Industry', RAND Journal of Economics, 3(2), 294-325).

15. For more discussion about market power in re-dispatch and nodal systems see Harvey, S.M. and Hogan, W.W., 2000. 'Nodal and Zonal Congestion Management and the Exercise of Market Power', LECG LLC working paper. 
16. At least around the equilibrium consumption level. Loads capable of responding quickly to price signals can be treated as generators in the ancillary service markets.

17. Transmission charges are typically levied on the retailers and on generators. They are then passed on to the electricity consumers.

18. One could argue that if generators implement the rational bidding strategy discussed in Section 4.4 their profits would depend on their location, which would restore the correct incentives to efficiently selecting plant locations. We do not pursue this line of reasoning here, since we want to emphasise the differences between the re-dispatch system and the nodal system that are likely to result in practice as a consequence of the various imperfections and frictions that we assumed thus far.

19. Recall that we are neglecting here transmission losses.

20. The same logic would apply to flow-gate transmission rights. A merchant approach is not consistent with a congestion management system based on re-dispatch. With re-dispatch the market perceives an unlimited supply of transmission rights, even in the event of congestion. As a consequence, there is no market for the right to use the transmission system.

21. We abstract here from the issues involved in assessing the amount of additional transmission rights that a network upgrade allows to be issued.

22. A detailed characterisation of the institutional setting is not necessary here. For example, private investors could be allowed to ask the system operator to implement the upgrade on their behalf. They would pay for the upgrade and receive the corresponding endowment of transmission rights.

23. As we discussed in Section 4.3, a network upgrade will generally allow the system operator to create alternative sets of new transmission rights. In this case the sponsors would select their preferred set of transmission rights.

24. The upgrade's sponsors could withdraw some of the financial transmission rights that they are granted by the system operator from the market. However, that would not mitigate the impact of the excess supply of physical transmission caused by the upgrade on spot energy prices.

25. The criteria for exemption from third party access for new interconnectors are laid out in Article 17 of European Commission Regulation No. 714/2009. 